\title{
Low and middle altitude cusp particle signatures for general magnetopause reconnection rate variations: 1 . Theory
}

Article

Published Version

Lockwood, M. and Smith, M. F. (1994) Low and middle altitude cusp particle signatures for general magnetopause reconnection rate variations: 1 . Theory. Journal of Geophysical Research, 99 (A5). 8531-8553,. ISSN 0148-0227 doi: https://doi.org/10.1029/93JA03399 Available at https://centaur.reading.ac.uk/38817/

It is advisable to refer to the publisher's version if you intend to cite from the work. See Guidance on citing.

Published version at: http://dx.doi.org/10.1029/93JA03399

To link to this article DOI: http://dx.doi.org/10.1029/93JA03399

Publisher: American Geophysical Union

All outputs in CentAUR are protected by Intellectual Property Rights law, including copyright law. Copyright and IPR is retained by the creators or other copyright holders. Terms and conditions for use of this material are defined in the End User Agreement.

www.reading.ac.uk/centaur 
Central Archive at the University of Reading

Reading's research outputs online 


\title{
Low and middle altitude cusp particle signatures for general magnetopause reconnection rate variations: 1 . Theory
}

\author{
M. Lockwood ${ }^{1}$ \\ Rutherford Appleton Laboratory, Chilton, Didcot, Oxon, United Kingdom
}

\author{
M. F. Smith \\ Laboratory for Extraterrestrial Physics, NASA Goddard Space Flight Center, Greenbelt, Maryland
}

\begin{abstract}
We present predictions of the signatures of magnetosheath particle precipitation (in the regions classified as open low-latitude boundary layer, cusp, mantle and polar cap) for periods when the interplanetary magnetic field has a southward component. These are made using the "pulsating cusp" model of the effects of time-varying magnetic reconnection at the dayside magnetopause. Predictions are made for both low-altitude satellites in the topside ionosphere and for midaltitude spacecraft in the magnetosphere. Low-altitude cusp signatures, which show a continuous ion dispersion signature, reveal "quasi-steady reconnection" (one limit of the pulsating cusp model), which persists for a period of at least $10 \mathrm{~min}$. We estimate that "quasi-steady" in this context corresponds to fluctuations in the reconnection rate of a factor of 2 or less. The other limit of the pulsating cusp model explains the instantaneous jumps in the precipitating ion spectrum that have been observed at low altitudes. Such jumps are produced by isolated pulses of reconnection: that is, they are separated by intervals when the reconnection rate is zero. These also generate convecting patches on the magnetopause in which the field lines thread the boundary via a rotational discontinuity separated by more extensive regions of tangential discontinuity. Predictions of the corresponding ion precipitation signatures seen by midaltitude spacecraft are presented. We resolve the apparent contradiction between estimates of the width of the injection region from midaltitude data and the concept of continuous entry of solar wind plasma along open field lines. In addition, we reevaluate the use of pitch angle-energy dispersion to estimate the injection distance.
\end{abstract}

\section{Introduction}

We discuss the theory of low and mid-altitude observations of cusp particle precipitation for time-dependent reconnection at the dayside magnetopause. We employ the "pulsating cusp" concept of Smith and Lockwood [1990] and restrict our attention to the cusp during southward interplanetary magnetic field (IMF) conditions.

The term "cusp" originates from the form of the geomagnetic field. In the paper that first predicted the existence of the magnetosphere, Chapman and Ferarro [1931] noted the existence of two magnetic null lines (which they termed "horns"). Precipitation of magnetosheathlike plasma near the ionospheric foot of these field lines was first found by Heikkila and Winningham [1971], who termed the precipitation cusp and postulated that the particle entry was possible because of the magnetic nulls. Further observations subsequently revealed magnetosheath plasma precipitated over a large fraction of the sunward edge of the polar cap, not just at a point, as would be predicted by entry around the

'Also at Imperial College, London, United Kingdom.

Copyright 1994 by the American Geophysical Union.

Paper number 93JA03399.

0148-0227/94/93JA-03399\$05.00 magnetic null. Heikkila [1972] termed the latter, more extensive, precipitation the "cleft", a topological term for the boundary of the closed field line torus (see review by Siscoe [1987]). Thus he assumed that these particles precipitated from a closed low-latitude boundary layer. For many years the terms cusp and cleft were used almost interchangeably for the precipitation, but no precise quantitative distinction between them was devised until that by Newell and Meng $[1988,1989]$. It should be noted that both terms come from magnetic field line topology and hence have inherent assumptions about the origins of the precipitating particle populations.

Since the discovery of cusp and cleft precipitations, evidence has steadily accumulated that magnetosheath plasma gains access to the magnetosphere as a result of the reconnection of the magnetosheath field with the geomagnetic field [Dungey, 1968; Frank, 1971; Reiff et al., 1977; 1980], and not because of the magnetic field minimum at the magnetic cusp. This evidence is varied and complex and is outlined briefly in the following four subsections.

\subsection{Accelerated Ion Flows}

Hill and Reiff [1977] noted that some cusp/cleft particles were energized to above magnetosheath energies, consistent with entry along convecting open field lines which form a 
rotational discontinuity (RD) at the dayside magnetopause. The production of such a convecting $\mathrm{RD}$ is, by definition, the process of magnetic reconnection. Because of the velocity filter effect, the accelerated ions will tend to be seen at the edge of the cusp which is closest to the magnetic reconnection separatrix. The existence of accelerated ions in cusp/cleft precipitation has recently been confirmed by Newell and Meng [1992] and Woch and Lundin [1992b]. The latter showed that they were on the equatorward/poleward edge of the cusp for southward/northward IMF, consistent with reconnection at low-latitude/lobe sites, respectively.

Magnetopause data also show that magnetosheath plasma enters the magnetosphere by flowing along open field lines. For an ideal-MHD RD the plasma should flow toward and away from the magnetopause at the local Alfvén speed and hence obey the Whalén relation. This has been confirmed by a large number of studies using the "stress balance test," both on the dayside [Aggson et al., 1983; Paschmann, 1985; Paschmann et al., 1979, 1986, 1990; Sonnerup et al., 1981, 1990; Gosling et al., 1990a; Maynard et al., 1991; Smith and Rodgers, 1991] and at the tail lobe boundary [Sanchez et al., 1990; Sanchez and Siscoe, 1990].

Cowley [1982] predicted that magnetosheath particles entering the magnetosphere along convecting open field lines, via an ideal-MHD RD, would show characteristic D-shaped distribution functions. These have recently been observed in data from a variety of spacecraft; ISEE 1 [Gosling et al., 1990b, c], AMPTE-UKS [Smith and Rodgers, 1991] and AMPTE-CCE [Fuselier et al., 1991]. Fuselier et al. used the mass resolution of the $\mathrm{CCE}$ ion detector to show that all populations from all sources were found to be as predicted by Cowley, on both sides of the magnetopause. In addition, Smith and Rodgers used the stress balance test to show that the low-energy cut off of the D-shaped distribution was the de-Hoffman Teller reference frame velocity, as also predicted by Cowley.

\subsection{Asymmetric Convection}

At the dayside magnetopause [e.g., Gosling et al., 1990a], at midaltitudes [e.g., Burch et al, 1985] and in the ionosphere [e.g., Lockwood and Smith, 1989], the injected magnetosheath plasma in the cusp is observed on eastward/westward convecting flux tubes for strongly negative/positive IMF $B_{y}$ in the northern hemisphere, and in the converse direction in the southern hemisphere. Given that the origin of this dawn-dusk asymmetry of dayside polar cap convection is the tension force on newly opened field lines [Atkinson, 1972; Jorgenson et al., 1972; Cowley, 1981], the large $\left|B_{y}\right|$ conditions reveal the cusp precipitation to be on recently opened field lines produced by dayside reconnection. The field-aligned currents bounding the cusp/cleft precipitation confirm this conclusion [Taguchi et al., 1993].

\subsection{Velocity Filter Effect}

The well-known energy-latitude dispersion of cusp ions during southward IMF was first explained by Rosenbauer et al. [1975] in terms of the velocity filter effect. This dispersion is sometimes also seen in cleft precipitation and extends into the ionospheric region termed "mantle." This has two important consequences. First, it means that field lines are convecting through the cusp region, which is as predicted by the reconnection model. Second, the range of precipitating ion energies reveals a range of injection locations as the field line convects [Onsager et al., 1993; Lockwood and Smith, 1993a]. Lockwood and Smith have pointed out that this range of locations is large and extends over the entire dayside magnetopause. This too is consistent with reconnection, for which magnetosheath plasma streams continuously across the magnetopause once the field line is open [Cowley et al., 1991b]. The broad entry region revealed by the velocity filter effect proves that the magnetic field minimum in the magnetic cusp is not the cause of the particle entry.

\subsection{Variation of Cusp Location with IMF Orientation}

Statistical studies have shown that the equatorward edge of the cusp migrates to lower magnetic latitudes when the IMF turns southward [Burch, 1973; Carbury and Meng, 1988]. This is consistent with the erosion of the magnetosphere by magnetic reconnection and places the cusp on newly opened field lines. Furthermore, the cusp is observed to move in local time, depending upon the sense and magnitude of the IMF $B_{y}$ component [Candidi et al., 1989; Newell et al., 1989]. This is predicted by the reconnection model because of the effects of asymmetric addition of open flux [Cowley et al., 1991a].

\subsection{Effects of Nonsteady Reconnection}

Recently, there has been interest in the behavior of the cusp/cleft precipitation when the reconnection rate is not steady. For over a decade, transient bursts of magnetopause reconnection have been invoked as a cause of transient particle and field signatures near the dayside magnetopause, which were therefore termed "flux transfer events" (FTEs) [Russell and Elphic, 1978, 1979; Haerendel et al., 1978; Paschmann et al., 1982; Berchem and Russell, 1984; Rijnbeek et al., 1984; Farrugia et al., 1987a, 1988; Southwood et al., 1986]. Lockwood and Smith [1989] noted that the flows, inferred filamentary field-aligned currents, and the precipitating ion and electron characteristics were consistent with the cusp as a whole being the ionospheric signature of an FTE (rather than the cusp representing a signature of steady reconnection, embedded within which would be much smaller FTE signatures). Prior to this a similar suggestion had been made by Menietti and Burch [1988], based on the width of the injection region that they inferred from midaltitude cusp ion observations; this width estimate is discussed further in section 5.2 of the present paper. From the occurrence frequency of the cusp precipitation, Newell [1990] objected that the extended longitudinal width of the statistical cusp (covering several hours of magnetic local time, MLT) was not consistent with the cusp being a nearly-circular signature of a fux transfer event, as predicted by Southwood [1985; 1987] for the Russell and Elphic [1978; 1979] FTE model. In reply, Lockwood and Smith [1990] pointed out that the longitudinal elongation could be consistent with an FTE, provided one adopts either the Southwood et al. [1988 and Scholer [1988] single elongated $\mathrm{X}$ line model, or alternatively the Lee and $\mathrm{Fu}$ [1985] multiple elongated X line model.

The pulsating cusp model [Smith and Lockwood, 1990] reproduces the occurrence statistics of cusp precipitation [Smith et al., 1992] and predicts the existence of jumps in the 
spectrograms of cusp ion precipitation at low altitudes [Smith et al., 1992; Cowley et al., 1991b]. These predictions were made using the Cowley and Lockwood [1992] theory of ionospheric convection. This model was developed to explain the observed responses of ionospheric convection to IMF changes [Lockwood et al., 1986; Etemadi et al., 1988; Todd et al., 1988; Lockwood et al., 1990; Saunders et al., 1992], but also successfully explains at least some dayside auroral and flow transients [Lockwood et al., 1993b, c]. It is based on the concepts put forward by Siscoe and Huang [1985] and Freeman and Southwood [1988]. Independent of the predictions by Cowley et al., Newell and Meng [1991] published examples of cusp ion spectrograms from the DMSP-F7 satellite which show exactly the predicted jump feature, but used a more spatial, rather than temporal, interpretation. Lockwood and Smith [1992] have used one of these examples and shown that the reconnection was pulsed, with virtually no reconnection taking place between the pulses. The ambiguity between spatial and temporal descriptions of these jumps, and of the cusp in general, arises from the motion of low-altitude satellites. This ambiguity was recently resolved by Lockwood et al. [1993a] who combined DMSP satellite observations of cusp ion jumps with coincident observations of poleward moving structures by the European Incoherent Scatter (EISCAT) radar. Such structures are predicted by the pulsating cusp model but not by steady spatial explanations. A further distinction is that the latter does not allow any flow across the boundary marked by the cusp ion jump: hence flows on both sides should be in the same direction, which was found not to be the case. These observations provide direct evidence for the pulsating cusp model and allowed Lockwood et al. to show that the lowest energy cusp ions (at any one observation time) were injected from near the subsolar magnetopause. This places the reconnection site at low latitudes on the dayside magnetopause, consistent with a number of studies of the dayside magnetopause when the IMF is southward [Gosling et al., 1990a; Paschmann, 1984; Berchem and Russell, 1984; Rijnbeek et al., 1984; Daly et al., 1984].

For low-altitude spacecraft, only cusp/cleft ions with very small pitch angles can be observed, all others having mirrored in the converging field before reaching the satellite. At higher altitudes, however, a range of pitch angles are observed. Menietti and Burch [1988] used the pitch angleenergy dispersion to estimate that the cusp ions originated from the magnetic cusp (see also Burch et al. [1986]). This is therefore in disagreement with the results of Lockwood et al. [1993a] and the magnetopause observations indicating subsolar reconnection and particle entry, as discussed above. In addition, Menietti and Burch estimated that the injection region was only $l R_{E}$ in extent, which also appears to contradict the reconnection model which predicts that particles stream continuously into the magnetosphere once a field line is opened. In section $\mathbf{5}$ of this paper, we reevaluate the use of midaltitude cusp ion observations to estimate the width of the injection region and the distance to the reconnection site and reconcile these two sets of apparently contradictory results.

In addition, the pulsating cusp model is frequently dismissed because of the observations of cusp particles over extended periods at middle altitudes and the high occurrence probability of the cusp precipitation a low altitudes [e.g., Newell and Sibeck, 1993]. In this paper, we use a simple model to demonstrate that magnetosheath plasma enters the magnetosphere along open field lines all the time that the field line is open and that particles reach the ionosphere, with densities and energies that classify them as cusp, for of order $10 \mathrm{~min}$. after the field line is reconnected (section 4). Because the precipitation persists for much longer than most intervals between FTE bursts (as deduced from magnetopause signatures), the persistence and high occurrence probability of the cusp precipitation, at either low or middle altitudes, are shown not to place any limits on how pulsed the dayside reconnection was (section 6). In order to achieve this we first need to discuss the particle signatures of time-varying reconnection at the magnetopause (section 3), in the topside ionosphere (section 4) and at middle magnetospheric altitudes (section 5). The reasons why the signatures at these three altitudes are so different are discussed in section 2.

\section{Effects of Spacecraft Altitude}

The altitude of a spacecraft determines the type of signature that will be observed near the dayside open/closed field line boundary when the magnetic reconnection at the magnetopause is not steady. This is because the relative velocity of the satellite and any particle/field signatures will have different causes at different altitudes. To a first-order approximation, two limits of the general behavior apply to observations at the dayside magnetopause and in the topside ionosphere (discussed briefly in sections 2.1 and 2.2 below, and in detail in sections 3 and 4 , respectively), whereas a more complex general case applies at middle magnetospheric altitudes (sections 2.3 and 5). A further complication is that the magnetic field is easily compressed at the magnetopause and, to a lesser extent, at midaltitudes. At low altitudes, however, the field is effectively incompressible. At all three locations there are three velocities which are important: the satellite velocity, $\underline{\mathrm{V}}_{s}$, the plasma convection velocity, $\underline{\mathrm{V}}_{\boldsymbol{r}}$, and the velocity of the open/closed field line boundary, $\vec{V}_{\mathrm{b}}$. All these velocities are here discussed in a frame of reference which is fixed with respect to the Earth.

\subsection{Magnetopause Spacecraft}

A satellite at the dayside magnetopause will, typically, have a boundary normal speed of the order of $V_{s}=2 \mathrm{~km} \mathrm{~s}^{-1}$ (in the Earth's frame of reference) [e.g., Hapgood and Lockwood, 1993], whereas compressional changes (by solar wind dynamic pressure changes) and erosion (by magnetic reconnection) of the boundary will cause boundary speeds, $V_{b}$, of typically $50 \mathrm{~km} \mathrm{~s}^{-1}$ (deduced from dual-satellite observations) [e.g., Russell and Elphic, 1978; Farrugia et al., 1989]. In addition, the open field lines produced by reconnection convect away from the reconnection $\mathrm{X}$ line at field-perpendicular convection speeds, $V_{c}$, of the order of $40 \mathrm{~km} \mathrm{~s}^{-1}$ [Aggson et al., 1983; Sonnerup et al., 1990; Gosling et al., 1990a; Paschmann et al., 1990; Maynard et al., 1991]. Note that the speed of field line motion along the magnetopause (the de-Hoffman Teller velocity) is $V_{F}=$ $V_{d} / \sin \theta_{s p}$, where $\theta_{s p}$ is the angle that the magnetospheric field makes with the boundary; that is, $\sin \theta_{s p}=B_{d} / B_{s p}$. Hence, for example, a (large) boundary normal field of $B_{n}=7.5 \mathrm{nT}$ [Sonnerup et al., 1990], a magnetospheric field at the dayside magnetopause of $B_{s p}=75 \mathrm{nT}$, and a convection velocity of $V_{c}=40 \mathrm{~km} \mathrm{~s}^{-1}$ yield a speed along the boundary of $V_{F}=$ $\left(B_{s p} / B_{n}\right) V_{c}=400 \mathrm{~km} \mathrm{~s}^{-1}$. The satellite speed is negligible in 
this case, and structures produced by time-varying reconnection will be moved over the satellite (by convection and by boundary motions): possible signatures which result are discussed briefly in section 3 .

\subsection{Low-Altitude Spacecraft}

At low altitudes $(h-1000 \mathrm{~km})$ the satellite speed in the

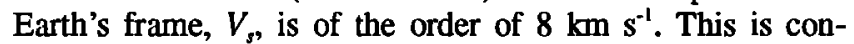
siderably greater than the ionospheric convection speeds which are $V_{c}-1 \mathrm{~km} \mathrm{~s}^{-1}$. In addition, ground-based observations indicate that the boundary between open and closed field lines moves in latitude [Horwitz and Akasofu, 1977; Foster et al., 1980; Lockwood et al., 1993b]; the most rapid motions being equatorward following a burst of magnetopause reconnection and have typical speeds of a degree of latitude per minute; that is, $V_{b}-2 \mathrm{~km} \mathrm{~s}^{-1}$. Hence the dominant motion is that of the spacecraft, which flies through the boundary and any structure on newly opened field lines. In this sense, low-altitude observations are an opposite limit to those at the dayside magnetopause. Low-altitude cusp particle signatures for general variations of the magnetopause reconnection rate are discussed in section 4.

\subsection{Midaltitude Spacecraft}

In the midaltitude cusp region, neither of the above limits fully apply. For example, a spacecraft at apogee of geocentric distance $r=3-4 R_{E}$, has a speed of $V_{s}-5 \mathrm{~km} \mathrm{~s}^{-1}$. Convection speeds $V_{c}$ are larger, being of the order of $10-50 \mathrm{~km} \mathrm{~s}^{-1}$ [e.g., Menietti and Burch, 1988]. No dual-satellite observations of boundary motions at this altitude are available, but simple mapping of magnetopause and ionospheric boundary motions would imply that $V_{b}$ is comparable to $V_{c}$. Hence such midaltitude observations are much more like those at the dayside magnetopause, in that the satellite speed is the minor contributor to the relative motion of the satellite and any signature of time-varying reconnection. However, from the above figures we find the ratios $\left(V / V_{s}\right)$ and $\left(V_{b} / V_{s}\right)$ may be in the range $2-10$, which is roughly an order of magnitude lower than the corresponding magnetopause values (section 2.1). Hence satellite motion cannot be fully neglected, unlike for the magnetopause observations. Furthermore, for satellites such as DE 1 and Viking when at altitudes between apogee and perigee, $V_{c}, V_{b}$, and $V_{s}$ can all be comparable.

\section{Magnetopause Signatures of Nonsteady Magnetopause Reconnection}

Figure 1 shows schematically the structure of the magnetopause predicted for reconnection taking place at a stationary and persistent $X$ line but with reconnection rate showing a pulsed enhancement over a background level. The $X$ line is to the left. Figure 1 is in the de-Hoffman-Teller reference frame, in which the field lines are at rest: this frame moves at velocity $V_{F}$ along the magnetopause, away from the $\mathrm{X}$ line, in the Earth's frame. This structure is predicted by the Southwood et al. [1988] and Scholer model of FTE signatures, based on a suggestion by Saunders [1983] and Biernat et al. [1987]. Similar conclusions have resulted from a

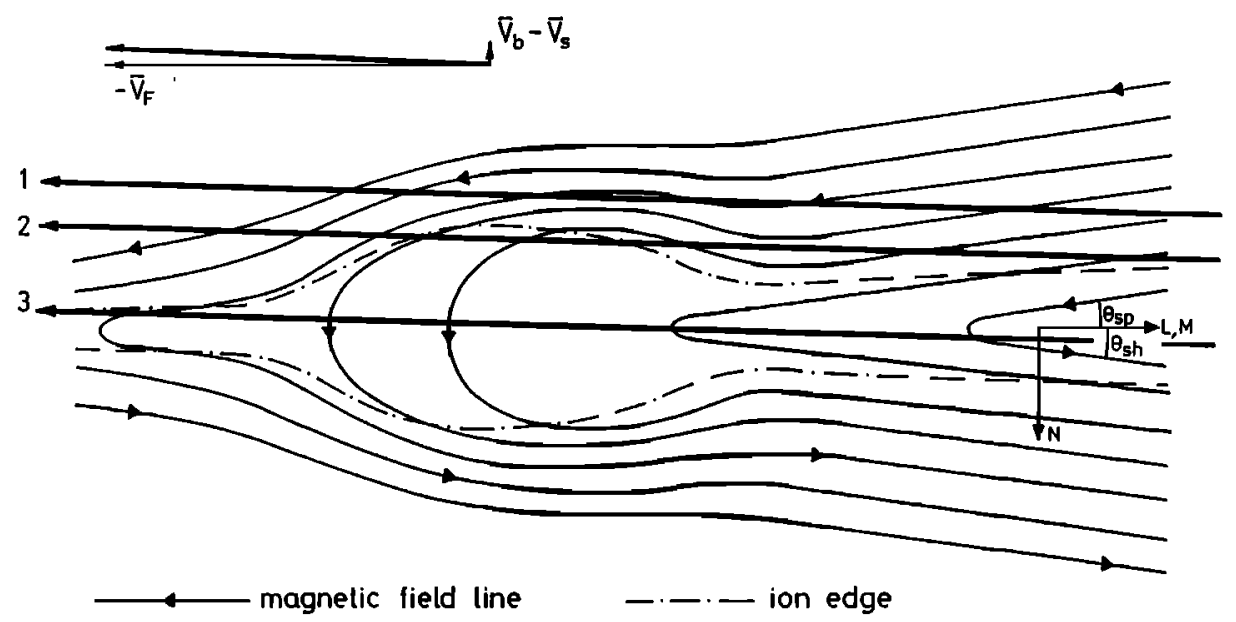

Figure 1. Magnetopause signatures of time-varying magnetopause reconnection. Open magnetic field lines, produced by a reconnection $X$ line to the left of the figure, are shown in two dimensions and in the de-Hoffman-Teller reference frame. The magnetopause is the rotational discontinuity (RD) of each magnetic field line at the $L M$ plane (the center of the diagram). The $N$ axis is the outward normal to the magnetopause, and hence the top half of the diagram is the magnetosphere, the lower half the magnetosheath. The magnetosheath and magnetospheric fields make angles $\theta_{s h}$ and $\theta_{s p}$ with the magnetopause. Also shown are three possible satellite paths: in this frame of reference the satellite moves to the left along the magnetopause at the de-Hoffman-Teller speed $V_{F}$ and inbound at velocity $\left(V_{b}-V_{s}\right)$, where $V_{b}$ and $V_{s}$ are the boundary velocity and the $N$ component of the satellite velocity, both measured in the Earth's frame and both positive in the $+N$ direction (outward). The dotted-dashed lines are the ion edges, between which accelerated ions are seen in the Earth's frame of reference. A pulse of enhanced reconnection rate (here over a nonzero background value) produces a region where the normal magnetic field and the tangential electric field at the magnetopause are enhanced, causing the angles of the $\mathrm{RD}, \boldsymbol{\theta}_{s h}$ and $\theta_{s p}$, to be greater. This produces a bulge in the ion edges and the draping signature in both magnetospheric and magnetosheath fields. 
variety of methods: Southwood et al. [1988] presented conceptual modeling; Scholer [1988] obtained similar results by two-dimensional MHD numerical simulations; and Semenov et al. [1992] have presented an analytic derivation. A simple physical understanding of this FTE model is obtained by applying the laws of conservation of energy and matter to the inflow and outflow regions of the reconnection $\mathrm{X}$ line. This shows that the field line outflow speed along the magnetopause, $V_{F}$, is determined by the Alfvén speeds in the inflow regions and is independent of the reconnection rate $E_{T}$. However, outside the diffusion region, $V_{F}$ is also equal to $\left(E_{T} / B_{n}\right)$, and hence an increase in $E_{T}$ causes a proportional increase in the boundary-normal magnetic field, $B_{n}$.

The pulse of reconnection produces a bulge in the reconnection layer, as shown in Figure 1, which yields the classic FTE magnetic signatures (a bipolar boundary normal field variation accompanied by a rise in the total field) as the structure moves over the satellite. Within this bulge the loops of newly opened flux produced by the reconnection burst thread the magnetopause. The newly opened flux and the bulge as a whole convect along the magnetopause away from the $\mathrm{X}$ line, at speed $V_{F}$. Note that the presence of reconnection before and after the burst means that there is open flux threading the boundary on either side of the traveling enhancement in the boundary normal field component. The dot-and-dash line is the "ion edge," as observed in magnetopause data [Gosling et al., 1990b]: on the magnetospheric side this marks the point where the most energetic magnetosheath ions have reached as the field lines convect away from the $X$ line. Likewise on the magnetosheath side the ion edge marks the boundary of the magnetospheric ions that have crossed the magnetopause. Within these edges the satellite will observe the accelerated ion flows [Paschmann et al., 1979; Gosling et al., 1990a].

In Figure 1 we consider three satellite paths through the structure. The satellite is moving inbound through the magnetopause at a speed $\left(V_{b}-V_{s}\right)$, where $V_{b}$ and $V_{s}$ are the outward boundary normal speeds of the magnetopause boundary and of the satellite, respectively, in the Earth's frame. The satellite also moves at speed $V_{F}$ toward the left of Figure 1 in the de-Hoffman-Teller frame. The three paths result from three different phasings of the reconnection pulse with respect to the satellite pass. For path 1 the satellite will detect the magnetic signature of a magnetospheric FTE but fail to see any accelerated ion flows. On the other hand, a satellite following path 2 will observe both accelerated flows and an FTE field signature. Notice that the accelerated ions are only observed in the region of the inward boundary normal field, and not in the prior period of outward field. This explains the observation that accelerated flows seen in association with FTEs are on the trailing edge of the events [Paschmann et al., 1982]. A spacecraft moving along path 3 would observe accelerated flows but would fail to detect an FTE signature. For such a path a pulse of additional boundary normal magnetic field $\left(B_{n}\right)$ and proportionally enhanced tangential electric field $\left(E_{T}\right)$ would pass over the satellite: however, because the boundary orientation cannot be determined with sufficient accuracy, these would remain undetected. A satellite passing along path 3 would be classified as observing "quasi-steady" reconnection.

The important conclusions are, first that the detection of accelerated ion flows and signatures of so-called quasi-steady reconnection do not in fact tell us about the reconnection rate, hence "quasi-continuous" is, strictly speaking, a better term than quasi-steady in this context. Second, the important consequence of the pulse of enhanced reconnection rate is the region of enhanced boundary normal field which convects away from the $\mathrm{X}$ line (the bipolar $B_{n}$ signatures, which have become termed as FTEs, are not in themselves important). It is possible that some FTEs signatures are, in fact, caused by surface waves on the magnetopause, as suggested by Sibeck [1990]; however, the observed dependence of magnetospheric FTE occurrence on magnetosheath field orientation shows that such cases are not common [Lockwood, 1991]. Conversely, bipolar $B_{n}$ signatures may not always be seen around a patch of enhanced boundary normal field. Hence in the remainder of this paper we discuss "pulsed reconnection" and reserve the term FTE for the characteristic set of magnetopause signatures, including the bipolar $B_{n}$ variation.

The magnetosheath particles cross the boundary continuously along all open field lines. On crossing the RD, all ions maintain a constant speed in the de-Hoffman-Teller reference frame (as, by definition, the electric field is zero in that frame) but are accelerated in the Earth's frame. This process gives rise to the D-shaped distribution functions of the injected ions. Figure 2 shows contours of the normalized ion distribution function, $f(v) / f_{\max }$, as predicted by Cowley [1982]. The magnetosheath ion temperature employed is $2 \times 10^{6} \mathrm{~K}$. The velocities are as measured in the Earth's frame of reference. The lower cutoff of the field parallel ion velocity, $V_{\mid l}$, is the de-Hoffman-Teller frame velocity $V_{F}$ times the cosine of the angle that the magnetospheric field lines make with the magnetopause, $\theta_{s p}$. At the reconnection $X$ line, $\theta_{s p}$ is close to zero. The form of $f(v)$ shown in figure 2 has recently been observed by Gosling et al. [1990c], Fuselier et al. [1990] and Smith and Rodgers [1991]. Smith and Rodgers also employed the stress balance test to show that the lowspeed cutoff in $V_{\|}$was, as predicted by Cowley, close to $V_{F}$.

Cowley and Owen [1989] have provided a method for evaluating how a newly opened field line evolves over the dayside magnetopause. These authors noted the field line velocity $V_{F}$ is such that the field-aligned flow in the deHoffman-Teller frame is at the local Alfvén speed $V_{A}$, as required for an ideal MHD RD. This means that the magnetosheath flow component along the boundary (away from the $\mathrm{X}$ line), in the de-Hoffman Teller frame, is $V_{S H}{ }^{\prime}$ which is equal to $-V_{A} \cos \theta_{s h}$, where $\theta_{s h}$ is the angle the magnetosheath field makes with the magnetopause. Converting into the Earth's frame, in which the flow along the boundary is $V_{S H}:$

$$
V_{F}=V_{S H}+V_{A} \cos \theta_{\mathrm{sh}}
$$

where the flow speed $V_{S H}$ is approximately that given by a gas dynamic model of magnetosheath flow around the boundary. Figure 1 shows that the pulse of enhanced reconnection alters the values of $\theta_{s h}$ and $\theta_{s p}$, both being increased in the bulge of the reconnection layer. However, these angles are equal to $\sin ^{-1}\left(B_{n} / B\right)$ which, for $B$ of $75 \mathrm{nT}$ and a large $B_{n}$ of $7.5 \mathrm{nT}$ [Sonnerup et al., 1990], is 5.74, the cosine of which is 0.995 . Hence, even for such a large $B_{n}$, the minimum cutoff in $V_{\|}$(which equals $\left\{V_{S H}+V_{A} \cos \right.$ $\left.\theta_{s h} \mid \cos \theta_{s p}\right)$ is almost independent of the reconnection rate. This means that the spectra of particles crossing the magnetopause at any one point of the boundary is not significantly 


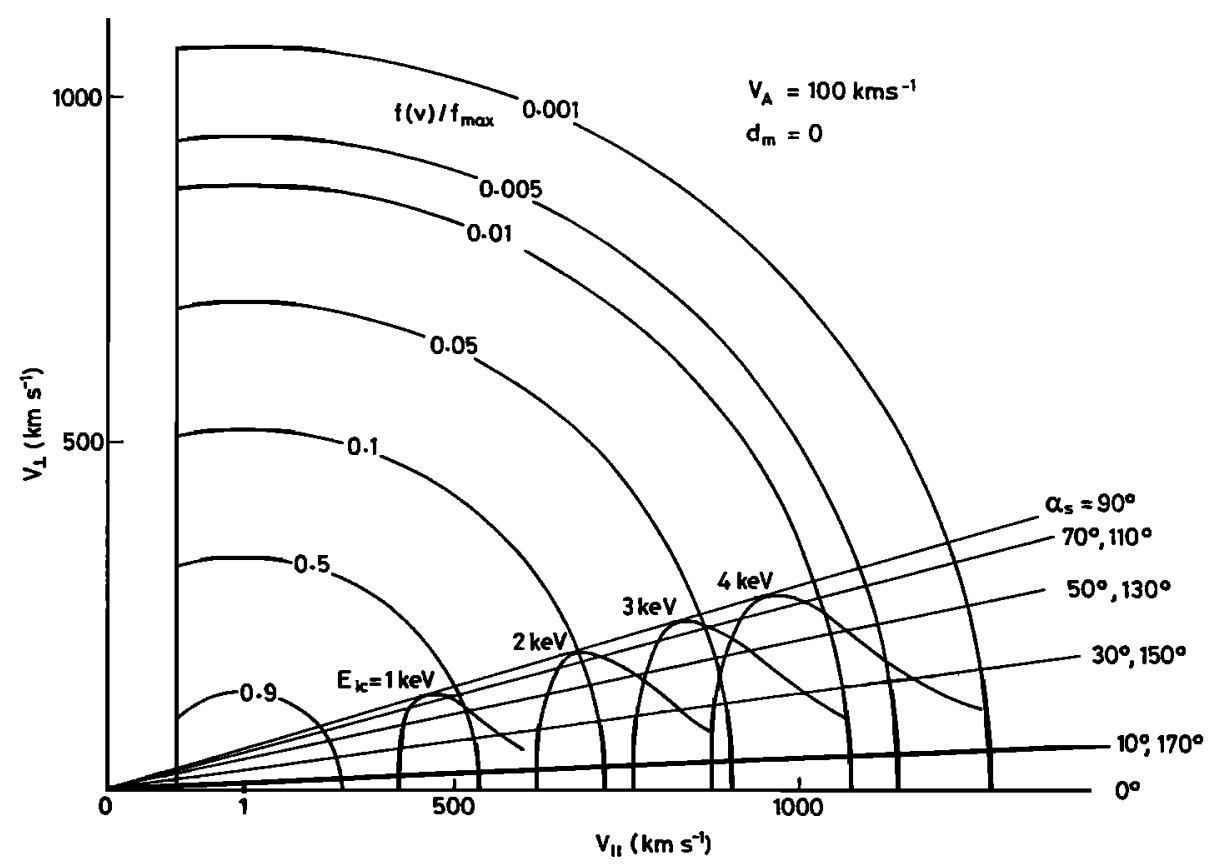

Figure 2. A distribution function $f(v)$ of magnetosheath ions injected across the $\mathrm{RD}$ at the dayside magnetopause (see Figure 1), as predicted by Cowley [1982]. The ion gas just outside the magnetopause is at a temperature of $2 \times 10^{6} \mathrm{~K}$, the Alfvén speed at the magnetopause is $100 \mathrm{~km} \mathrm{~s}^{-1}$ and (for a location close to a subsolar $\mathrm{X}$ line, $d_{m}=0$ ) the angle $\theta_{s p}$ is very close to zero. Contours of $f(v)$ as a ratio of its peak value, $f_{\max }$, are shown as a function of field-parallel and field-perpendicular components of ion velocity $\nu$. Also shown are lines of constant pitch angle, $\alpha_{s}$, as observed by a satellite at geocentric height $r_{s}=3.98 R_{E}\left(1 R_{E}=1\right.$ mean Earth radius): the pitch angle folding being calculated using the T87 magnetic field model (see text for details). The four curves connect ions of equal time of flight to the satellite, each curve being classified by the minimum ion energy at zero pitch angle $\left(\alpha_{s}=0\right), E_{i c}$.

influenced by the rate of reconnection at the time when the field line was opened.

Most of the ions of the distribution function shown in GFigure 2 do not reach middle altitudes. Those with pitch angle greater than about $15^{\circ}$ mirror in the converging magnetic field and are convected into the tail mantle. However, those with pitch angle less than about $15^{\circ}$ are observed at middle latitudes, and those with pitch angles very close to zero reach ionospheric altitudes.

\section{A Simple Illustrative Model of Low-Altitude Signatures for General Reconnection Rate Variations}

We describe a model of cusp particle entry, acceleration, and precipitation as a newly opened field line evolves over the dayside magnetopause. We concentrate on the ion precipitation characteristics. This is because the energylatitude [Reiff et al., 1978] and the pitch angle-energy [Burch et al., 1982] dispersions show that ions undergo adiabatic, scatter-free motion from the magnetopause to the ionosphere. The same cannot be said, in general, of the electrons. As pointed out by Burch et al. [1982], Onsager et al. [1993], the electrons (with magnetosheath densities) would be seen closer to the open/closed field line boundary than the ions if they too simply moved in such a manner. Sometimes some electrons are seen equatorward of the ion edge at midaltitudes, and they display pitch-angle energy dispersion (electron V's) [Burch et al., 1982], but usually the cusp ions and electrons (of comparable density) have roughly the same equatorward edge [Newell and Meng, 1988; Newell et al., 1989; Onsager et al., 1993]. This means that the cusp ion motions do influence those of the lower-momentum electrons, presumably via small ambipolar electric fields and in such a way so as to maintain the observed quasi-neutrality of the cusp [Burch, 1985].

\subsection{Model Description}

We make use of the gas-dynamic predictions of magnetosheath flow along the magnetopause, as presented by Sprieter et al. [1966]. These predictions are shown in Figure 3a. The top panel shows the ratio $V_{s H} / V_{s w}$, as a function of $d_{m} / d_{s}$, where $V_{S H}$ is the magnetosheath flow speed near the boundary, $V_{S W}$ is the undisturbed solar wind flow, and $d_{m}$ is the distance along the boundary from the nose of the magnetosphere (which is at a GSM $X$ coordinate of $d_{s}$ ). The predictions employed here are for a typical solar wind Mach number of 8 [Hapgood et al., 1991]. The remainder of Figure 3 shows some other parameters that are input into the model and not computed in a self-consistent manner. Figure $3 \mathrm{~b}$ gives the field-aligned distance from each point on the magnetopause to the ionosphere, $d_{1}$ (also as a ratio of $d_{s}$ ). This distance is based on mappings using the Tsyganenko T87 magnetic field model from the ionosphere to close to the nominal magnetopause location. This variation of $d$ is only regarded as illustrative because the T87 model does not allow for open magnetic flux threading the dayside magnetopause [Crooker et al., 1991]. The important feature is this: as an open field line evolves away from the subsolar reconnection 


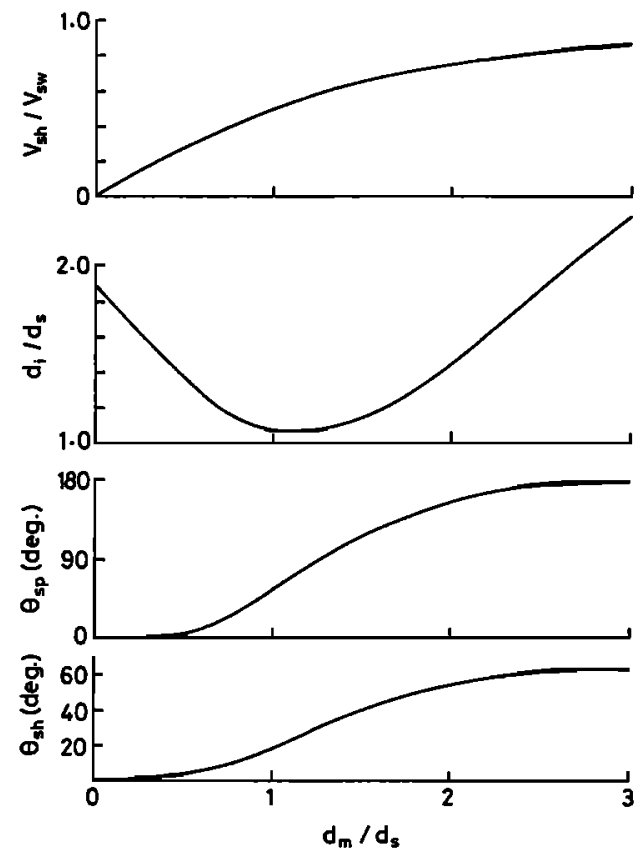

Figure 3. Inputs to a model of cusp ion precipitation, shown as a function of $\left(d_{n} / d_{s}\right)$, where $d_{m}$ is the distance from the subsolar point, which is at $\left(d_{s}, 0,0\right)$ in GSE coordinates. All these illustrative inputs are taken, as a first approximation, to be independent of reconnection rate. The top panels shows the magnetosheath flow speed around the boundary, $V_{s h}$, normalized to the speed of the upstream solar wind $V_{s w}$ from the gas-dynamic predictions of Sprieter et al. [1966] for solar wind Mach number 8 . The second panel shows the distance from the magnetopause to the ionosphere (as a ratio of $d_{s}$ ) adopted from the T87 magnetic field model. The lower two panels shows the RD angles $\theta_{s h}$ and $\theta_{s p}$ (see Figure 1).

point, the distance $d_{i}$ falls as it approaches the magnetic cusp, but subsequently increases again as it is carried into the tail lobe. The final two panels show example variations of the angles of the RD at the magnetopause, $\theta_{s h}$ and $\theta_{s p}$. These angles are taken to be a function of position only, and hence any variations of these angles with boundary normal field (as discussed in section 3) are neglected. The variation of the angle $\theta_{s p}$ adopted gives a value of zero at the subsolar magnetopause, $90^{\circ}$ at the magnetic cusp and approaches $180^{\circ}$ as the open field line is swept into the tail lobe. The actual variation of $\theta_{s h}$ will depend strongly on the orientation of the undisturbed IMF impinging upon the bow shock. The adopted variation is intended to reflect the draping of field lines for an IMF vector which points in the -Z(GSM) direction. For simplicity, the Alfvén speed at the boundary, $V_{A}$, is assumed to be independent of $d_{m}$, and values of 100 and $400 \mathrm{~km} \mathrm{~s}^{-1}$ are employed with $V_{s w}=400 \mathrm{~km} \mathrm{~s}^{-1}$, in order to calculate the field line speed over the boundary, $V_{F}$, using (1). We here take $d_{s}$ to be $10 R_{E}$.

The assumptions inherent in these inputs mean that each newly opened field line evolves into the tail lobe in an identical manner, under the actions of magnetic tension and magnetosheath flow and independent of variations in the rate of reconnection. From the inputs given in Figure 3 we can use (1) to compute the evolution of a newly opened field line over the dayside magnetopause, as a function of time elapsed since it was reconnected. Figure 4a shows the variation of the normalized distance from the nose to the point where the field line threads the boundary $\left(d_{m} / d_{s}\right)$ as a function of $\left(t-t_{0}\right)$, where $t_{o}$ is the time when that field line was reconnected. The dashed line is for the higher $V_{A}$ case of $400 \mathrm{~km} \mathrm{~s}^{-1}$, the solid line for $100 \mathrm{~km} \mathrm{~s}^{-1}$. Figure $4 \mathrm{~b}$ shows the variation of $V_{F}$. In the low Alfvén speed case the field line evolves only slowly away from the $X$ line, reaching the magnetic cusp $\left(\theta_{s p}\right.$ $\left.=90^{\circ}\right)$ at $\left(t-t_{0}\right) \approx 375 \mathrm{~s}$ and subsequently approaching the undisturbed solar wind speed $V_{S W}$ asymptotically. For the higher Alfven speed the field line accelerates away from the reconnection site rapidly under the influence of magnetic tension and reaches a peak speed near the magnetic cusp at $\left(t-t_{0}\right) \approx 150 \mathrm{~s}$. It subsequently slows to $V_{S w}$.

As discussed in section 3, the minimum speed of the injected distribution is $V_{F} \cos \theta_{s p}$. The corresponding minimum ion energy $E_{\min }$ is plotted in Figure 4c. Initially, it increases with time as the field line accelerates, but at the magnetic cusp it falls to zero, because there $\theta_{s p}$ is $90^{\circ}$.

Onsager et al. [1993] have recently applied Loiuville's theorem to a complimentary model to that described here. Their model is more rigorous in that it predicts the latitudinal variation of the full cusp ion and electron spectra, using the gas-dynamic predictions of the magnetosheath density and temperature as a function of $d_{m}$. To achieve this, they adopted fixed models for the electric field and magnetic field in the dayside magnetosphere. Here we take a somewhat different approach and restrict our attention to the low- and high-energy cutoffs of the ion spectrum seen in the ionosphere. The advantage of this approach is that we can discuss the time-dependent case, and it is not necessary to assume how the electric field maps down the open field lines (Onsager et al. in fact assume it to be constant). The latter is a particular problem for the time-dependent cases, where induction effects are significant.

We term the lowest ion energy observed at any one time and place the "cutoff ion energy" $E_{1 c}$ and the maximum detectable energy $E_{m t^{\prime}}$. Because confusion can arise between the times of ion injection and observation, we denote the latter by $t_{s}$ (the subscript $s$ for satellite). $E_{m d}$ will be a function of instrument sensitivity. We here adopt values appropriate to the DE 2 ion detector, LAPI [Winningham et al., 1981], which we will employ in a companion paper (Lockwood and Smith, manuscript in preparation 1994). If the minimum distribution function we can detect is $f\left(V_{t h}\right)$, we will record ions of (thermal) speeds up to $V_{t h}$ in the plasma rest frame which, by the Cowley [1982] theory, is a speed of $\left(V_{A}+V_{t h}+V_{F} \cos \theta_{s p}\right)$ in the Earth's frame. We term the energy corresponding to this speed $E_{\max }$. Note $E_{i c}$ and $E_{m d}$ are the limits of the spectrum observed by the satellite at time $t_{s}$ (on a field line which was reconnected at time $t_{0}$ ), whereas $E_{\min }$ and $E_{\max }$ are the limits of the spectrum injected (and accelerated) at the magnetopause at time $t$. Time-of-flight effects mean that there is no simple relationship between these two pairs of energies $\left(E_{i c}, E_{m d}\right.$ and $\left.E_{\min }, E_{\max }\right)$.

To estimate $V_{\text {tor }}$ we note that LAPI typically detects cusp ions with $f(v)$ down to about $10^{-3}$ of the peak of the distribution function, $f_{\max }$ (Lockwood and Smith, manuscript in preparation, 1994). We then estimate $V_{t h}$ for a Maxwellian distribution of plasma temperature (assumed isotropic) predicted by the gas-dynamic model. The variation of $E_{\max }$ with time elapsed since reconnection is shown in Figure 4d: there is a more modest increase in $E_{\max }$ (than for $E_{\min }$ ) as the field line approaches the magnetic cusp. $E_{\max }$ then falls as the 

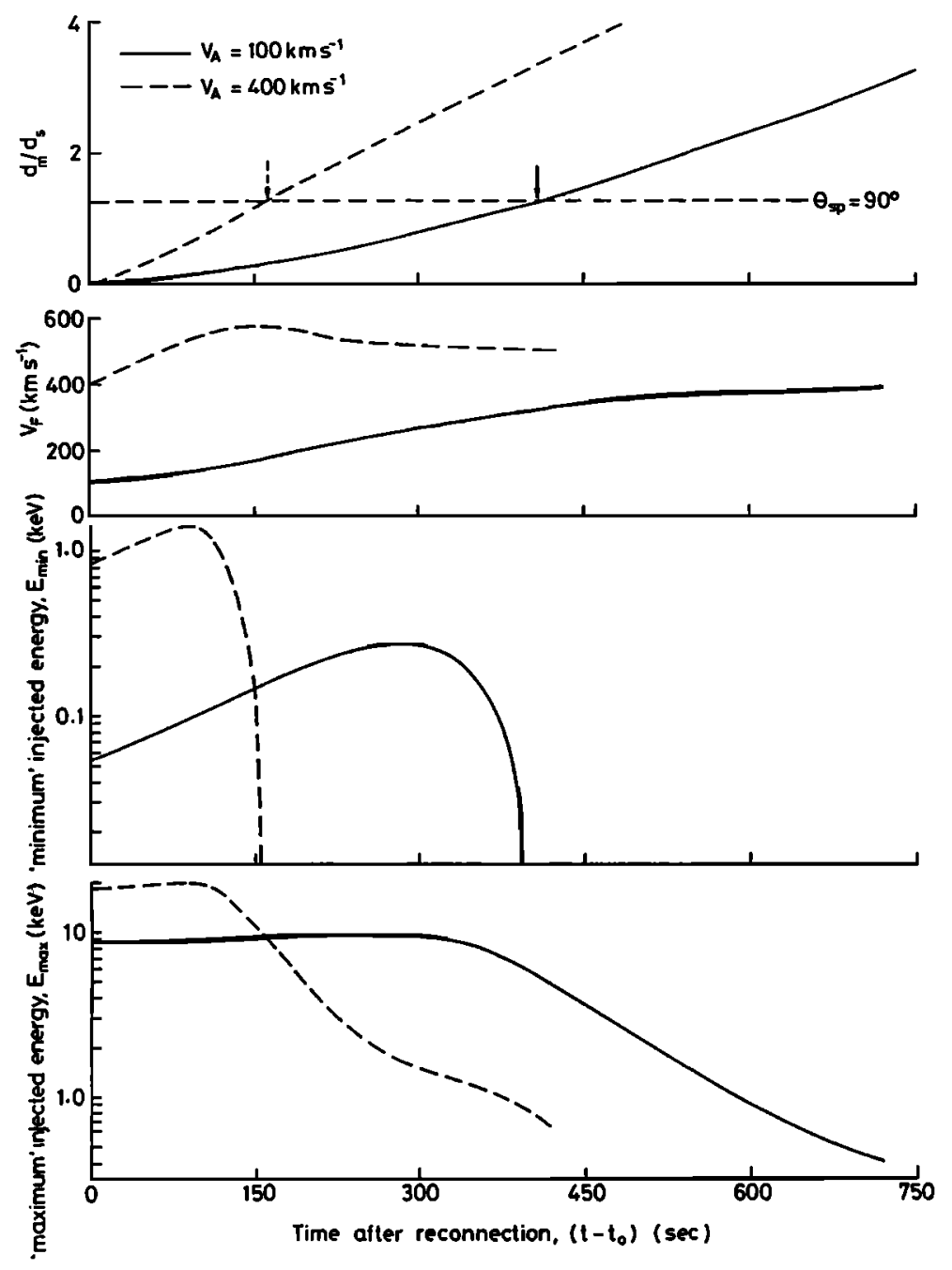

Figure 4. Predictions of the model of magnetosheath ion injection. As a function of time elapsed since the field line was reconnected $\left(t-t_{0}\right)$ are shown: the normalised distance of the injection point (where the field line threads the boundary) from the subsolar $\mathrm{X}$ line $\left(d_{m} / d_{j}\right)$; the de-Hoffman-Teller velocity, $V_{F}$; and the minimum and maximum observable energy of the magnetosheath ions injected at that time, $E_{\min }$ and $E_{\max }$. Solid curves are for a magnetosheath Alfvén speed of $100 \mathrm{~km} \mathrm{~s}^{-1}$, dashed curves are for $400 \mathrm{~km} \mathrm{~s}^{-1}$. The arrows in the top panel mark the time that the field line reaches the magnetic cusp, as defined by $\theta_{s p}$ $=90^{\circ}$.

field line passes through the magnetic cusp, and the ion acceleration turns to deceleration (i.e., at latitudes above magnetic cusp $\left(\theta_{s h}+\theta_{s p}\right)>\pi$ and J.E $<0$ [Hill and Reiff, 1977; Hill, 1979].

The times of arrival in the ionosphere of injected ions of energy $E_{\min }$ and $E_{\max }$ are then calculated for every point on the magnetopause, through which the newly opened flux tube passes as it evolves toward the tail lobe. For each time elapsed since reconnection $\left(t_{s}-t_{0}\right)$, we determine the lowest $E_{\operatorname{mnn}}$, which is $E_{i c}$, and the largest $E_{\max }$ which is $E_{\operatorname{me}}$. Hence we determine the maximum and minimum ion energy seen by the satellite at an observation time $t_{s}$, irrespective of when and where on the magnetopause those ions were injected. In fact, as noted by Lockwood and Smith [1992], the ions of energy $E_{i c}$ originate from the reconnection $\mathrm{X}$ line (provided $E_{i c}>E_{\min }$ for the location of the $\mathrm{X}$ line), whereas those of energy $E_{m d}$ are injected nearer the magnetic cusp.

The results are shown in Figure 5, which shows $E_{i c}$ and $E_{m d}$ as functions of time elapsed since reconnection, for the two magnetopause Alfvén speeds of $100 \mathrm{~km} \mathrm{~s}^{-1}$ (solid curves) and $400 \mathrm{~km} \mathrm{~s}^{-1}$ (dashed curves). The ion spectrum detected by the satellite would lie between these two curves for each $V_{A}$. The curves show an evolution of the spectrum, as predicted by Cowley et al. [1991b] and also numerically computed by Onsager et al. [1993]. The precipitation evolves continuously from (open) cleft/LLBL to cusp to mantle to polar cap as the field line convects, this evolution was recently confirmed from combined satellite and radar data by Lockwood et al. [1993a].

The value of the Alfvén speed has a number of interesting consequences. For the higher Alfvén speed the ions injected on the dayside are more highly accelerated. This means that higher-energy ions are observed, and these arrive earlier than the first ions seen for the low Alfvén speed case. However, because the newly opened field line evolves faster over the dayside magnetopause, it reaches the magnetic cusp earlier and the maximum injected ion energy falls sooner because the ion acceleration turns to deceleration sooner. Hence the 


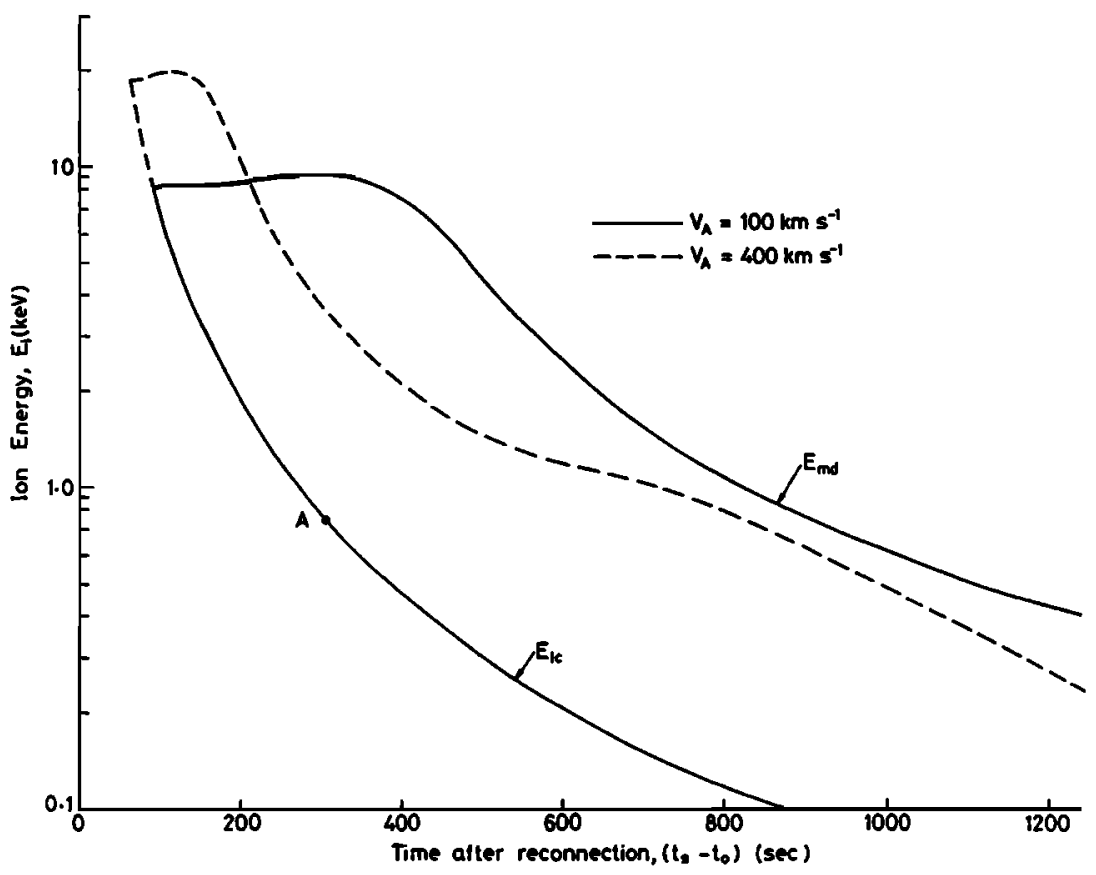

Figure 5. Predicted ion precipitation at low altitudes for the model described in Figures 3 and 4 . The minimum and maximum ion energy observed in the ionosphere, $E_{i c}$ and $E_{m b}$ are shown as a function of the "age" of the open field line; that is, the field line observation time $\left(t_{t}\right)$ minus the time when that field line was reconnected $\left(t_{o}\right)$. The solid curve is for a magnetosheath Alfvén speed of $100 \mathrm{~km} \mathrm{~s}^{-1}$, the dashed curve is for $400 \mathrm{~km} \mathrm{~s}^{-1}$.

width of the ion spectrum is less than for the low Alfven speed case. This difference decreases as the field line evolves because in both cases the field line speed approaches that of the undisturbed solar wind as it enters the lobe.

The Alfvén speed also has a important consequence for the lower cutoff ion energy. For the $V_{A}=100 \mathrm{~km} \mathrm{~s} \mathrm{~s}^{-1}$ case the lowest-energy ions (for the range of energies shown) all originated from the dayside $X$ line. However, for the higher Alfvén speed case this is not so for elapsed times greater than that of the point $A$ in Figure 5. This is because the field lines move away from the $\mathrm{X}$-line at speed $V_{A}$, and hence there are no ions injected here with velocity (in the Earth's frame) less than $V_{A}$. The point $A$ is at an energy of $833 \mathrm{eV}$, which corresponds to this minimum ion speed of $400 \mathrm{~km} \mathrm{~s}$. (In fact, a similar point exists for the $V_{A}=100 \mathrm{~km} \mathrm{~s}^{-1}$ case, but this is at $52 \mathrm{eV}$, which is below the range of energies shown.) At times greater than that of the point $A$ the lowestenergy ions observed were not injected at time $t_{0}$ and at the $X$ line, but at a later time, when the field line has evolved to higher latitudes and the ion acceleration has decreased.

\subsection{Effects of Steady and Time-Varying Reconnection}

As discussed in section 2 , the dominant velocity in the intersection of a low-altitude satellite with the cusp is that of the satellite. If the magnetic reconnection takes place at a steady rate, a low-altitude satellite passing through the cusp would see field lines of successively larger/smaller elapsed time since reconnection, as it approaches/moves away from the merging gap on open field lines; that is, there would be a smooth increase/decrease of $\left(t_{s}-t_{0}\right)$ with satellite observation time $t_{s}$. In both cases the satellite would observe a cusp ion spectrogram with minimum and maximum ion energies as in
Figure 5, where the $\left(t_{o}-t_{s}\right)$ axis could be replaced by distance from the last closed field line (the scaling factor depending on the satellite speed and on the angle of the satellite path with respect to the merging gap normal). This axis could also be replaced by observation time $t$, for a satellite that is moving away from the merging gap and by $-t_{s}$, for one that is approaching it. Such examples are observed (e.g., Figure 1 of Newell et al. [1989], Figure 2 of Onsager et al. [1993]). From Figure 5 we can estimate that for the lower cutoff ion energy $E_{i c}$ to fall smoothly to about $100 \mathrm{eV}$, requires the reconnection to have been steady for a period of about $\mathbf{1 0}$ min. Therefore such examples are direct evidence that reconnection can be quasi-steady for periods of at least 10 min, and we will discuss what this means in terms of a fluctuation level of the reconnection rate later in this section.

Figure 6a shows schematically the evolution of one field line, which was reconnected at a time $t_{o}$, as a function of time elapsed since reconnection (each position is labeled by the value of $\left(t-t_{0}\right)$ in minutes). If the dayside reconnection and convection were in a steady state, a snapshot of the open field lines at one time $t$ would be identical to Figure 6a (each field line having a different reconnection time $t_{o}$ ). However, if the reconnection is pulsed, this snapshot would not be the same, even if Figure 6a remains a valid description of how each open field line evolves. Figure $6 b$, is a snapshot at a time $t$, for which the reconnection has been pulsed with a squarewave waveform (pulses last $2 \mathrm{~min}$ and are separated by intervals $5 \mathrm{~min}$ long when the reconnection rate is zero). Each pulse produces a patch on the magnetopause of boundary normal magnetic field (and tangential electric field) where the boundary is an $\mathrm{RD}$, and these patches evolve away from the reconnection site. Between these patches, the boundary is a tangential discontinuity (TD). A low-altitude 


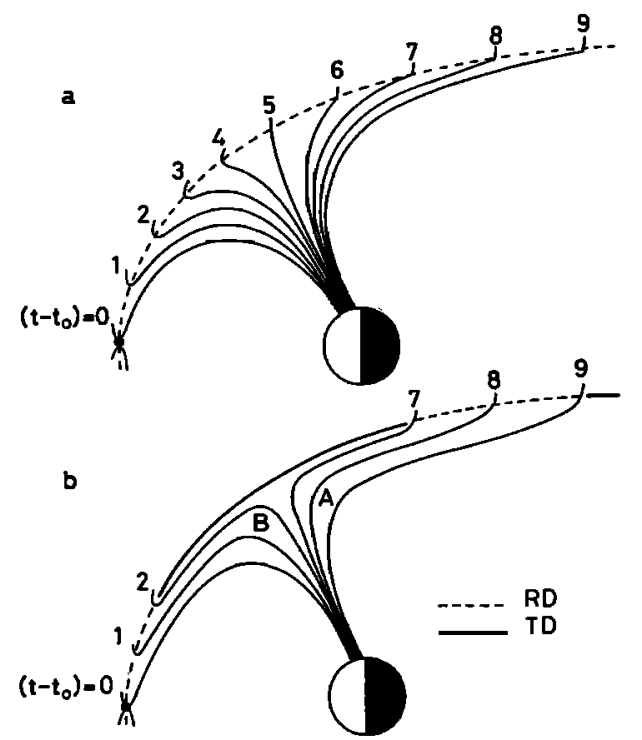

Figure 6. Schematic illustration of the effects of pulsed reconnection on magnetospheric topology. (a) The evolution of any one open field line which was reconnected at a time $t_{o}$, labeled by the time $t$. For steady-state conditions this is also a snapshot of all dayside open field lines at a time $t$, each field line being labeled by it reconnection time $t_{0^{*}}$ (In both cases the elapsed time since reconnection is $\left.\left(t-t_{o}\right)\right)$. (b) A snapshot at time $t$ for the case of entirely pulsed reconnection with reconnection taking place in the intervals 0-2 $\mathrm{min}$ and 7-9 $\mathrm{min}$ before the time $t$, giving rise to two distinct regions of open field lines labeled B and A, respectively. Open segments of the magnetopause (RD) are shown as dashed, segments where the boundary normal field is zero (TD) are shown as solid lines.

satellite in Figure $6 a$ would see the quasi-steady cusp discussed above. However, a low-altitude satellite flying through the cusp in Figure 6(b) would see jumps in the cusp ion spectrogram because $\left(t_{0}-t_{s}\right)$ is not a smooth function of $t_{s}$. The ionospheric signatures corresponding to Figure $6 \mathrm{~b}$ were plotted by Cowley et al. [1991b] and Smith et al. [1992]. Examples of such jumps have recently been reported by Newell and Meng [1992], Escoubet et al. [1992] and Lockwood et al. [1993a]. A method for evaluating the reconnection rate variation from the cusp ion spectrograms, by inverting the theory of Cowley et al. [1991b], has been developed by Lockwood and Smith [1992] and reveals that the reconnection can, at least sometimes, be entirely pulsed, as in Figure 6b. We conclude that both the quasi-steady (over periods up to about $10 \mathrm{~min}$ ) and completely pulsed situations can exist. It should be emphasized that the pulsating cusp model can be applied to any reconnection rate waveform, from a constant value (the steady state limit) to discrete pulses of any shape with absolutely no reconnection taking place between them [Smith et al., 1992]. Hence the pulsating cusp model is a generalisation, not a contradiction, of the steady-state concepts of the cusp as used by Rosenbauer et al. [1975], Reiff et al. [1977, 1978], and Hill and Reiff [1978].

To illustrate the effects of a pulsed reconnection rate, we use the waveform in Figure 7. The period of the variation is $8 \mathrm{~min}$, the average value inferred from magnetopause FTE

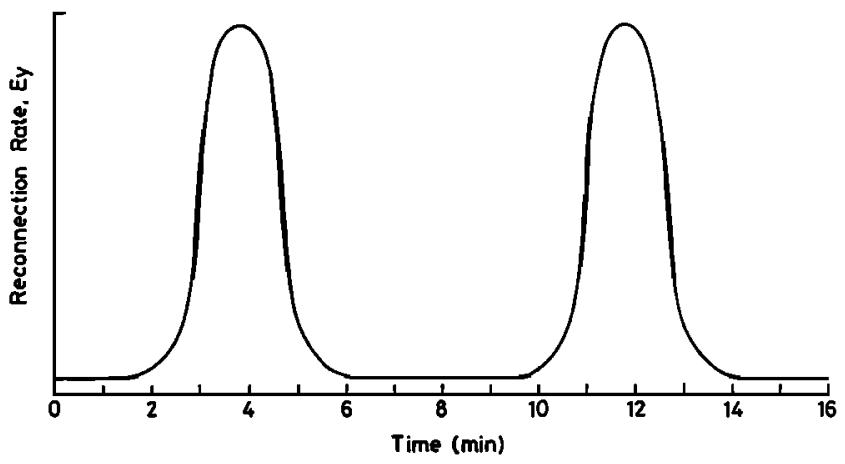

Figure 7. Model variation of reconnection rate $E_{y}$. The pulses repeat every $8 \mathrm{~min}$ and the background reconnection rate between the pulses is a fraction $a / b=0.04$ of the peak value of each pulse.

observations [Rijnbeek et al., 1984; Berchem and Russell, 1984]. (In section 6 we will make allowances for the fact that this period shows a broad distribution of values, up to about $30 \mathrm{~min}$, with a mode value of $3 \mathrm{~min}$ [Lockwood and Wild, 1993]). Between the pulses, the reconnection rate is here taken to fall to a much smaller, but nonzero, value. Comparison of Figures $6 \mathrm{a}$ and $6 \mathrm{~b}$ illustrates that the field lines between the magnetopause and the ionosphere do differ for a given $\left(t-t_{o}\right)$ and hence the distance $d_{1}$ will, in general, be dependent on the variation of reconnection rate. Here we neglect this effect on $d_{i}$, in the same way that we do not allow for corresponding effects on the RD angles, $\theta_{s h}$ and $\theta_{s p}$. Proper allowance for these effects will require full numerical MHD modelling of the dayside magnetosphere, with pulsed anomalous resistivity introduced at the reconnection $X$ line, as done for local simulations of the magnetopause reconnection layer by Scholer [1988].

Using the equations given by Lockwood and Smith [1992], we can convert $\left(t_{s}-t_{0}\right)$ and the merging gap electric field $E_{y}$ into the observation time $t_{s}$. The results are shown in Figure 8 for Alfvén speeds of $100 \mathrm{~km} \mathrm{~s}^{-1}$ (solid line) and $400 \mathrm{~km} \mathrm{~s}^{-1}$, as for the steady state case in Figure 5. The periods of low reconnection rate $\left(E_{y}\right)$ produce the steep gradients in $E_{i c}$ and $E_{\text {ma }}$ whereas the pulses of enhanced reconnection produce the smaller gradients. The appearance of the spectrogram is seen to be strongly dependent on the Alfvén speed. For $V_{A}$ $=400 \mathrm{~km} \mathrm{~s}^{-1}$ the jumps in $E_{m d}$ are as large as those in $E_{t c} ;$ an example of such a "staircase" cusp ion spectrogram has been presented by Escoubet et al. [1992]. However, the steps in $E_{m d}$ for the $V_{A}=100 \mathrm{~km} \mathrm{~s}^{-1}$ case are much smaller than those in $E_{i c}$ and resemble those presented, for example, by Lockwood et al. [1993a]. Indeed, for the lower Alfvén speed case, it is even possible for $E_{m d}$ to jump up when $E_{i c}$ jumps down.

The jumps in Figure 8 are less steep than those predicted by Cowley et al. [1991] and Smith et al. [1992], simply because the input model reconnection rate does not go to zero between the pulses. Figure 9 illustrates the effect on a jump in $E_{\imath c}$ of a steady background rate of reconnection (on which the pulses are superposed). We consider square wave pulses of reconnection rate, $E_{p}$ of amplitude $b$ and with a steady background reconnection rate of $a$ (as shown in Figure $9 a)$. When the ratio $a / b$ is unity the reconnection is steady; 


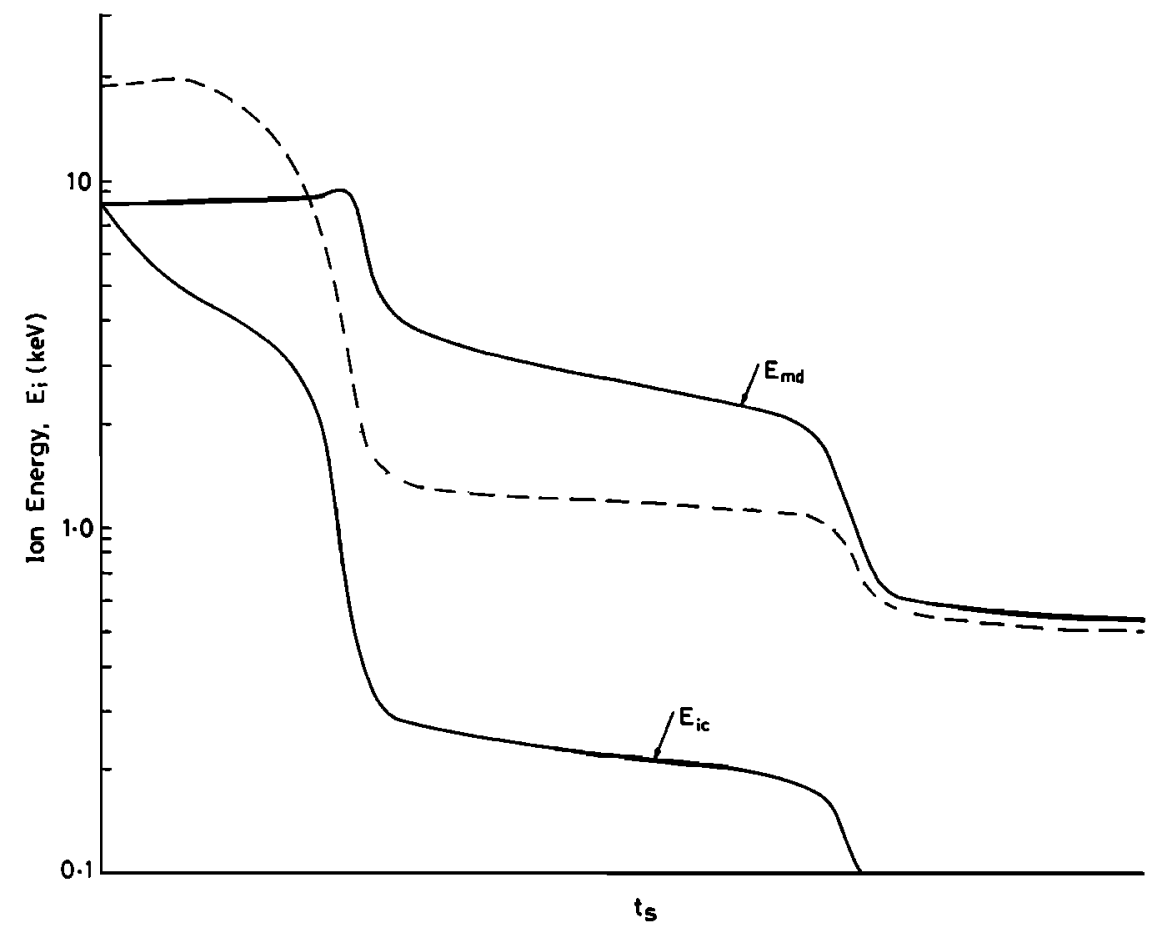

Figure 8. Predicted effect on cusp ion observations at low altitudes of pulsed magnetopause reconnection. The minimum and maximum observable cusp ion energies, $E_{k c}$ and $E_{m b}$ are shown as a function of the time of observation, $t_{s}$. Conditions are as for Figure 5, with the variation of reconnection rate with time as given in Figure 7. The solid curves are for a magnetosheath Alfvén speed of $100 \mathrm{~km} \mathrm{~s}^{-1}$, the dashed curves are for $400 \mathrm{~km} \mathrm{~s}^{-1}$.

when $a / b$ is zero the reconnection only takes place in the pulses. In addition to showing the resulting jumps in $E_{i c}$ for these two limits in Figure 9b, we also show those for two intermediate $a / b$ ratios $(0.67$ and 0.33$)$. In all four cases the interval between the pulses is $350 \mathrm{~s}$. For $a / b=0$ a clear discontinuity in the $E_{c c}$ variation is observed (and any other changes in the spectrum, for example that in $E_{m b}$ will similarly be discontinuous). As $a / b$ approaches unity, the jump in the spectrogram becomes increasingly difficult to discern. This yields a crude estimate how steady the reconnection rate is for those cusp spectrograms which we described earlier as showing quasi-steady reconnection. We believe changes in slope of the magnitude shown for $a / b=$ 0.33 may just have elicited some attention, but those for $a / b$ $=0.67$ would almost certainly go unnoticed. We would therefore interpret so-called quasi-steady reconnection (as inferred from cusp spectrograms) as showing fluctuations in reconnection rate were smaller than a factor of about $2(a / b$ $>0.5$ ).

The jumps discussed in the literature thus far [Newell and Meng, 1992; Lockwood et al., 1993a; Escoubet et al., 1992] all reveal $a / b=0$; that is, there is a complete cessation of reconnection between each pulse, and hence they indicate that large portions of the dayside magnetopause will be a $\mathrm{TD}$, as shown in Figure $6 \mathrm{~b}$.

\section{Midaltitude Signatures}

As discussed in section 2, for increasing satellite altitude above the topside ionosphere, the satellite velocity $V_{s}$ is smaller and will become comparable to both the convection
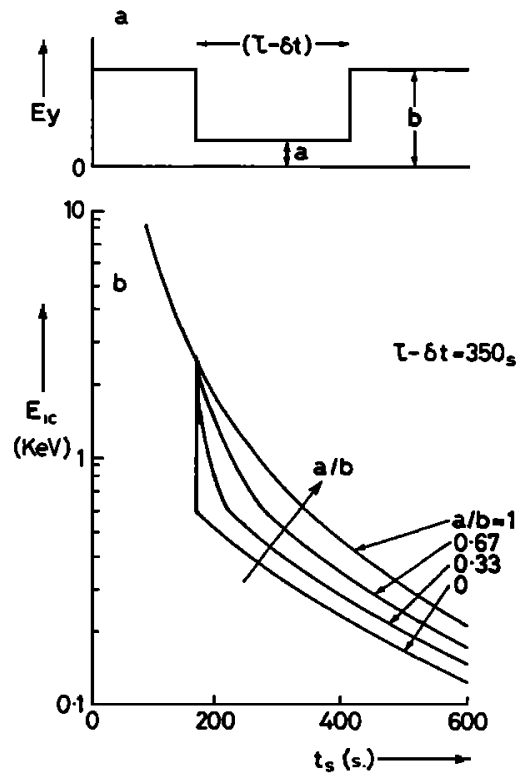

Figure 9. The effect on low-altitude cusp ion observations of background reconnection rate between pulses $a$. (a) Model (square) waveform of reconnection rate $\left(E_{y}\right)$ variations. (b) Curves of the minimum ion energy $E_{i c}$ as a function of observation time $t_{s}$ for four ratios of the background/peak ratio of the reconnection rate waveform, $a / b=1,0.67,0.33$ and 0 . In all cases the interpulse interval is $(\tau-\delta t)=350 \mathrm{~s}$. (The parameter $\tau$ is the pulse repeat period and $\delta t$ is the pulse duration). 
speed $V_{c}$ and the speed of the open/closed boundary motions due to both erosion and compression, $V_{b}$. At yet greater altitudes, $V_{s}$ becomes smaller than the other two velocities. In this section we discuss the increased complexity of the signatures which result if the magnetic reconnection rate is not steady. We consider all the velocities to be normal to the open/closed boundary. In a generalized three-dimensional case the relevant velocities will be the component normal to the open/closed boundary, provided that there are no variations along the boundary. Positive values are considered to be away from the open/closed boundary, on the open side of that boundary.

Figure 10 predicts the behavior of the minimum cusp ion energy $E_{\imath c}$. At midaltitudes this is the minimum energy observed at zero pitch angle, (i.e., at the point of the characteristic V form in ion spectrograms, see section 5.3). Variations of $E_{t c}$ with time of observation $\left(t_{s}\right)$ are shown for various cases when the reconnection is completely pulsed $(a / b=0)$. Figure $10 \mathrm{a}$ is for the special case when $V_{s}$ is identical to $V_{c}$, such that the satellite remains on the same open flux tube. In this special case the ion energy ramps down as it would for steady state conditions; that is, it is independent of the reconnection rate and its variations. In this case therefore the cusp ion spectrogram yields no information on the reconnection rate. In Figure 10b, $V, \gg$ $V_{c}$, as for a low-altitude satellite moving away from the open/closed field line boundary. This situation will be

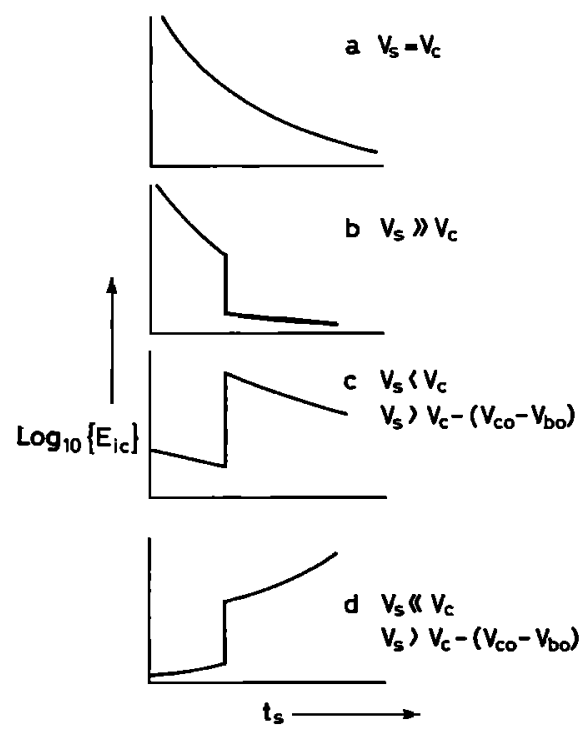

Figure 10. Variations of the lower cutoff ion energy $E_{i c}$ (at pitch angle $\alpha_{s}=0$ ), as a function of observing time $t_{s}$ as seen by a midaltitude spacecraft when the reconnection is completely pulsed $(a / b=0)$. (a) $V_{s}=V_{c}$; (b) $V_{s} \gg V_{c}$; (c) $\left(V_{c}\right.$ $\left.V_{c o}+V_{b o}\right)<V_{s}<V_{c}$; (d) $V_{s}<<V_{c}$ such that $\left(V_{c}-V_{c o}+V_{b o}\right)$ $>V_{s}$; where $V_{c}$ and $V_{s}$ are the convection and satellite speeds at the time the field line is observed $\left(t_{s}\right)$ and $V_{c o}$ and $V_{b o}$ are the convection and boundary speeds at the time the field line was reconnected $\left(t_{0}\right)$. All velocities are the component normal to the open/closed boundary and are positive away from the boundary on the open field line side. In Figure 10b the satellite moves from the region $B$ to the region A shown in Figure $6 \mathrm{~b}$., whereas in Figure $10 \mathrm{c}$ and $10 \mathrm{~d}$ the satellite moves from $A$ to $B$. The jump is due to the satellite motion in Figure 10b, the convection of the boundary in Figure 10c, and either/both in Figure 10d. relatively rare at middle altitudes, where generally $V_{s}<V_{c}$. Hence we would expect the form of cusp ion jumps seen at low altitudes (Figure 10b) to be rare at midaltitudes.

Figures 10c and 10d shows situations which will apply more frequently at midaltitudes (near apogee for satellites such as Viking and DE 1), where $V_{s}<V_{c}$. In such cases the convection carries a cusp ion energy jump over the satellite. For example, in Figure $6 \mathrm{~b}$ the satellite could initially be in region $A$ but convection moves region $B$ over it; hence in this example the satellite would initially be on a field line which was reconnected $7 \mathrm{~min}$ before the time of observation $\left(t_{s}-t_{o}=7 \mathrm{~min}\right)$, but the poleward convection of the field lines would move it onto one that was reconnected just $2 \mathrm{~min}$ before the time of observation $\left(t_{s}-t_{0}=2 \mathrm{~min}\right)$. As this happened, $E_{i c}$ would jump upward, irrespective of the direction in which the satellite is moving. The gradient of $E_{i c}$ away from this discontinuity depends upon the speeds $V_{s}, V_{c}$, and $V_{b}$. If the time of field line reconnection $t_{o}$ varies relatively slowly with distance $x$ (measured in the rest frame of the plasma and positive away from the open/closed boundary), such that the gradient of the time elapsed since reconnection with observation time obeys the inequality:

$$
\mathrm{d}\left(t_{s}-t_{o}\right) / \mathrm{d} t_{s}=1-\mathrm{d} t_{d} / \mathrm{d} t_{s}=1-\left(V_{s}-V_{c}\right)\left(\mathrm{d} t_{o} / \mathrm{d} x\right)>0
$$

then the observed $E_{c c}$ away from the upward jump will fall with time, as in Figure 10c. Were the left-hand side of (2) to be smaller than zero, then $E_{s c}$ would rise with time, as in Figure $10 \mathrm{~d}$. To understand the inequality (2), note that field lines further from the boundary (at greater $x$ ) were reconnected earlier (at lower $t_{o}$ ) and hence that $\left(\mathrm{d} t_{o} / \mathrm{d} x\right)$ is, by definition, negative and has a magnitude set by the reconnection rate. From Figure $1 \mathrm{~b}$ of Lockwood and Smith [1992], we note that $-\left(\mathrm{d} x / \mathrm{d} t_{o}\right)$ is equal to the speed with which the flux tube crossed the open/closed boundary when it was reconnected $\left(V_{c o}-V_{b o}\right)$, where the additional subscript $o$ denotes that these are the convection and boundary speeds when the field line was reconnected (at time $t_{0}$ ). Hence (2) can be rearranged to give

$$
V_{s}>V_{c}-\left(V_{c o}-V_{b o}\right)
$$

If this inequality holds, then the energy ramps down with time away from the jump. To evaluate the likelihood of this, we note that during a reconnection pulse the boundary erodes equatorward $\left(V_{b o}<0\right)$ [Lockwood et al., 1993b]. Hence, if the convection speed subsequently remains constant or increases $\left(V_{c}>V_{c o}\right)$, then (3) must apply to any satellite moving away from the merging gap $\left(V_{s}>0\right)$ (and it may also often apply to satellites moving toward it). However, at midaltitudes the boundary and convection speeds are both influenced by compressive motions of the magnetic field, as well as by reconnection, hence it is possible that (3) will not apply and the spectrogram will be as in Figure 10d. We conclude that (3) is likely to hold and hence that the signature shown in Figure 10c should be the most common midaltitude signature of pulsed reconnection. However, (3) may not always apply and the signature may sometimes be as in Figure $10 \mathrm{~d}$ and there could even be no signature, as in Figure 10a.

A second situation in which Figure 10d applies is for a low-altitude satellite which flies across the newly opened field lines and is approaching the open/closed boundary, as was discussed in the previous section. 


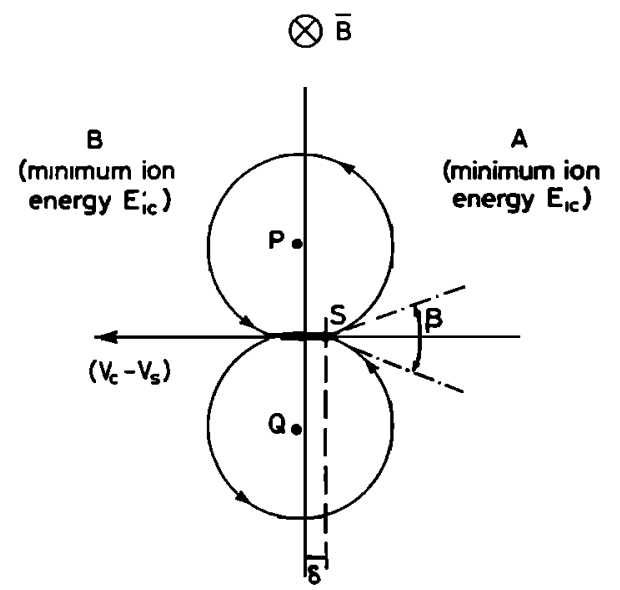

Figure 11. Midaltitude satellite observations close to the boundary of two regions of newly opened flux, A and B (see Figure $6 \mathrm{~b}$ ). This boundary is shown in the rest frame of the plasma, in which the satellite moves from $A$ to $B$ at speed $\left(V_{c}-V_{s}\right)$, where $V_{c}$ and $V_{s}$ are the velocities of convection and satellite motion normal to the open/closed boundary (both in the Earth's frame of reference). When at $S$, the satellite sees ions frozen onto the field lines of region $A$ but can also see large gyroradius ions which are frozen onto field lines on the other side of the boundary in region $B$ with guiding centers at, for example, $P$ and $Q$. The distance $\delta$ from the boundary over which this can occur depends on the azimuthal beam width of the detector $\beta$ and the gyroradius of the ions. At $S$, only field-aligned ions of region $A$ are observed.

\subsection{Midaltitude Ion Spectrograms}

Unlike at low altitudes, a midaltitude satellite observes a range of ion pitch angles which complicates the jump in $E_{i c}$. Figure 11 shows the path of the satellite across the boundary between two regions, such as $A$ and $B$ in Figure 6b. The figure is in the rest frame of the plasma, in which the satellite moves from the magnetic flux produced by the earlier reconnection pulse (region $A$ ) into that produced by the later pulse (region $B$ ) at a speed $\left(V_{c}-V_{s}\right)$. The satellite has a "cartwheel" motion (as for DE 1 and Viking), such that its spin axis is normal to the direction of motion. Hence as it spins, the ion detector viewing direction cycles continuously through four orientations: (1) $90^{\circ}$ pitch angle ions are observed by looking antiparallel to the direction of motion, then (2) precipitating field-aligned ions are observed by looking upward, out of the plane of the diagram, then (3) $90^{\circ}$ pitch angle ions are observed by looking in the direction of motion, and lastly (4) upward moving field-aligned ions are observed before completing the cycle. Figure 11 shows the azimuthal beamwidth of the detector, $\beta$, when in orientation 1. As it approaches the boundary, the satellite (at the location $S$ in Figure 11) will, in orientations 1 and 3, begin to observe large gyroradius ions whose guiding centers ( $Q$ and $\mathbf{P}$, respectively) are on field lines which were opened by the later of the two bursts (region B) whilst, in the same spin, it continues observe ions precipitating down the field lines (orientation 2) which were reconnected in the earlier burst (region A). As the satellite moves toward the boundary, the minimum gyroradius of ions from the other side of the boundary will decrease (and hence lower energy ions with pitch angles lower than $90^{\circ}$ will be seen). A similar finite-

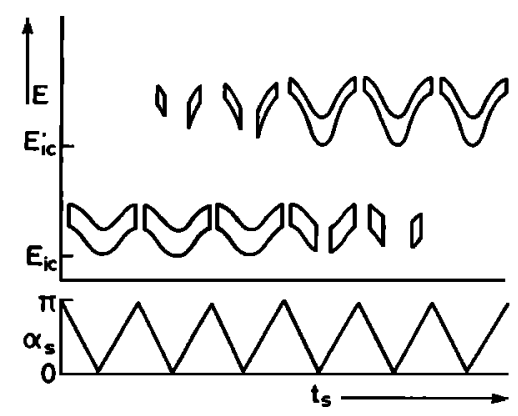

Figure 12. Schematic detail of a midaltitude cusp ion spectrogram, showing finite gyroradius effects around the jumps described in Figures $10 \mathrm{c}$ and 10d, where minimum ion energy jumps up from $E_{i c}$ to $E_{i c}{ }^{\prime}$ (as the satellite moves from region $A$ into region $B$ in Figure $6 b$ ). (top) $A$ contour of differential ion flux $J_{E}$ as a function of observing time $t_{s}$. (bottom) The pitch angle $\alpha_{s}$ observed by the spinning detector as a function of $t_{s}\left(\alpha_{s}=0\right.$ is the downward fieldaligned direction).

gyroradius effect would be seen after the satellite has crossed the boundary, but the minimum gyroradius of ions frozen onto the flux tubes on the other side of the boundary increases with time as the satellite moves away from the boundary.

Figure 12 demonstrates schematically a spectrogram which could therefore be seen around a jump of the kind predicted in Figure 10c and 10d (i.e., $V_{c}>V_{s}$ ). This figure shows one contour of differential energy flux in an energy-time spectrogram format for a spinning detector, such that the observed pitch angle $\left(\alpha_{s}\right)$ varies as shown in the lower panel. The lower cutoff ion energy jumps up from $E_{v c}$ to $E_{i c}^{\prime}$ as the boundary between regions $A$ and $B$ is convected over the satellite. This jump is considered instantaneous, such that the magnetopause is a TD between the two patches of boundary normal magnetic field (Figure 6b); hence Figure 12 is a midaltitude signature corresponding to the instantaneous jumps seen in cusp ion spectrograms at low altitudes [Lockwood and Smith, 1992; Escoubet et al., 1992; Lockwood et al., 1993a]. For the first three satellite spins shown in Figure 12 the detector sees full V-shaped signatures of ions [Burch et al., 1982, 1985; Menietti and Burch, 1988] injected onto field lines opened by the first of the two reconnection pulses (region A). The origin of the V-shaped signature is discussed in section 5.3. Consider the evolution of the spectrum of field-aligned ions. In Figure 12, for the first three times that the ion detector looks up the field line, away from the Earth $\left(\alpha_{s}=0\right)$, the minimum ion energy is $E_{i c}$; however, on the fourth it jumps up to $E_{v c}{ }^{\prime}$. This is because between spins 3 and 4 , the satellite has crossed between $A$ and $B$.

A key prediction of these finite gyroradius effects around the jump in the spectrogram is that the field-parallel ions of lower cutoff energy $E_{r c}$ are never observed at the same time as those with lower cut-off energy $E_{a c}{ }^{\prime}$ but that the former population must disappear on the same satellite spin as the latter population appears, and visa versa. For this to be caused by a double injection onto one field line the temporal separation of the two injections would have to be exactly equal to the difference in the ion times of flight. The probability of this coincidence is negligibly small, and hence the behaviour of the field-parallel ions provides a test for the above effect and hence for the existence of magnetopause TD 
separating patches of RD (i.e., for completely pulsed magnetopause reconnection). Note that ions with pitch angles nearer $90^{\circ}$ are seen simultaneously from both regions. The later paper (Lockwood and Smith, manuscript in preparation, 1994) describes an example of exactly the behavior in the ion spectrogram shown in Figure 12, while a conjugate lowaltitude spacecraft observes a cusp ion jump.

These finite gyroradius effects require the satellite to be very close to the boundary and hence that the satellite speed and the plasma convection speed be comparable, if the "overlapping ions" feature is to be observed for a prolonged period. From Figure 11 we find that the satellite must be within a distance $\delta$ of the boundary,

$$
\delta=\rho \sin (\beta / 2),
$$

where $\rho$ is the ion gyroradius and $\beta$ is the azimuthal acceptance angle of the ion detector. Hence the time that the overlapping populations are observed around the jump in $E_{i c}$ is

$$
d t=\left[\left(\rho+\rho^{\prime}\right) \sin (\beta / 2)\right] /\left(V_{c}-V_{s}\right)
$$

where $\rho$ and $\rho^{\prime}$ are the peak gyroradii on either side of the boundary. Considering $90^{\circ}$ pitch angle protons with $E_{1}$ of 1.0 $\mathrm{keV}$ and $E_{i}^{\prime}$ of $2.0 \mathrm{keV}$, we compute typical values for $\rho$ and $\rho^{\prime}$ of 4.6 and $6.5 \mathrm{~km}$, for a magnetic field of $1000 \mathrm{nT}$. For the DE 1 High-Altitude Plasma Instrument (HAPI), the azimuthal acceptance angle of each detector is $2.5^{\circ}$ (full width at half maximum) with a total acceptance angle of $\beta$ $=5^{\circ}$ [Burch et al., 1981]. Equation (5) shows that $\left(V_{c}-V_{s}\right)$ must be smaller than $0.02 \mathrm{~km} \mathrm{~s}^{-1}$ if $d t$ is to exceed $24 \mathrm{~s}$. (four 6-s spins, as in Figure 12). Such prolonged overlapping ion features will therefore be rare but could have three causes: first the satellite can be at an altitude where the magnitudes of $V_{s}$ and $V_{c}$ are comparable; second, the convection streamlines can be at close to $90^{\circ}$ to the satellite path; and third, the satellite may lie at the peak excursion, caused by a compression and subsequent expansion of the magnetosphere, of a cusp ion jump boundary. Prolonged overlapping features will obviously be less rare if entirely pulsed reconnection is common.

\subsection{Calculation of Width of Injection Region}

As mentioned in the introduction, there are two pieces of evidence which, at first sight, do not appear to agree with the theory of mid-altitude cusp signatures discussed in this section. These are the distance from the satellite to the injection point, and the width of the injection region, as deduced from midaltitude data. Menietti and Burch [1988] (hereinafter referred to as M\&B) derived this distance and width from, respectively, the pitch angle-energy dispersion (i.e., from the angle of the ion V's on the spectrograms) and the range of energies at a given pitch angle (i.e., the thickness of the V's) (see also Burch et al., [1986]). In the following subsection (5.3), we consider the calculation by M\&B of the injection distance; in this section we first consider their derivation of the width of the injection region.

M\&B estimated the spatial extent of the injection region to be roughly $1 R_{E}$. On this basis they suggested that the cusp and FTEs may be related. Initially, this appears to be inconsistent with the concepts presented here: once a field line is open, the plasma streams in continuously, as has been observed both on the dayside and in the tail lobe (see section 1.1)[Gosling et al., 1990; Sanchez and Siscoe, 1990; Sanchez et al., 1990; Phillips et al., 1993]. The only way that the inflow could be stemmed after the field line has moved just $1 R_{E}$ along the boundary is for the field line to be closed again and for it to remain closed.

The maxim discussed in section 4 for field-parallel ions will apply to any pitch angle, namely that the lowest-energy ions (largest time of flight) will have been injected at the reconnection site (provided their speed exceeds the Alfven speed at the boundary), whereas the highest-energy ions (lowest time of flight) for the same pitch angle will have been injected onto the same field line at a later time and hence at a location close to the magnetic cusp [Lockwood and Smith, 1992; 1993]. Cusp particles are injected continuously onto any one opened field line as it evolves over the dayside magnetopause, and hence the injection region should be at least $10 R_{E}$ in extent. It is true that a pulse of reconnection will result in a bundle of newly opened field lines, and from magnetopause FTE signatures we infer that these are typically $1 R_{E}$ in extent along their direction of motion [Farrugia et al., 1988] and in the boundary normal direction [Saunders et al., 1984]. However, these widths do not influence the precipitation characteristics on any one field line and neither is the extent which $M \& B$ estimate.

M\&B gained the estimate of $I R_{E}$ by two methods. However, in both cases they multiplied the field-perpendicular velocity by a time and hence estimated the width in the fieldperpendicular direction. This is only the same as the distance along the magnetopause near the magnetic cusp where the open field lines (on the magnetospheric side of the boundary) are roughly normal to the magnetopause $\left(\theta_{s p}=\pi / 2\right)$. At the dayside magnetopause (equatorward of the magnetic cusp) open field lines thread the boundary via the $\mathrm{RD}$, such that on the magnetospheric side of the boundary they make an angle $\theta_{s p}(<\pi / 2)$ with the magnetopause. Hence an element of width of the injection region along the magnetopause is a factor $1 / \sin \left(\theta_{s p}\right)$ larger than the corresponding field-normal element of width estimated by M\&B. Note that this factor tends to infinity near the reconnection site where $\theta_{s p}$ tends to zero. For example, for the RD with (large) $B_{n}$ of $7.5 \mathrm{nT}$ and $B_{s p}$ of $75 \mathrm{nT}, \mathrm{M} \& \mathrm{~B}$ 's estimate would correspond to a distance along the magnetopause of about $10 R_{E}$ (and greater for smaller $B_{n}$ ).

From analysis of an observed example (Lockwood and Smith, manuscript in preparation), we find that this is the major reason why the estimates of the width of the injection region by $\mathrm{M} \& \mathrm{~B}$ are of the order of $1 R_{E}$ when cusp particles actually cross all points of the dayside magnetopause along open field lines. In addition, there are a number of other factors which all cause the M\&B's width to be a slight underestimate. In both methods employed by M\&B the convection speed observed at the satellite was mapped to the magnetopause, with the assumption of steady state for a dipole field (their equation (4)): in other words, induction effects were ignored, and these will result in an underestimation of the field-perpendicular magnetopause convection velocity for pulsed reconnection [Lockwood and Cowley, 1992] and hence in the width of the injection region.

One method employed by M\&B is based on the length of time for which the V's detected have the peak ion energy observed (see their Figure 4). This assumes that the fall of 
the peak ion energy reflects a time-of-flight effect after cessation of injection. However, the reconnection theory shows that the peak injected ion energy falls as the field line convects because the acceleration of the particles as they cross the RD decreases [Hill and Reiff, 1977; Reiff, 1978]. This decay in the acceleration can be caused by the decrease in the field line velocity but is usually due to the straightening of the RD (Figure 4). Hence a fall in peak energy is not caused by a cessation of injection and a time-of-flight effect, as assumed by $M \& B$, but by the decay of the acceleration and a time-of-flight effect. This factor leads to a further underestimation of the width.

The other method of M\&B employed the range of ion energies seen at any one time or, more precisely, the width of the $V$ as seen in the differential energy flux spectrogram. However, the width of an ion $\mathrm{V}$ will depend upon the time elapsed since reconnection (as does the separation of $E_{m d}$ and $E_{i c}$ for the field-aligned case - see Figure 5). Hence if anything other than a low-energy $\mathrm{V}$ is used (large timeelapsed since reconnection), the full width of the $V$ and hence of the injection region will be underestimated. In addition, the minimum ion energy at larger pitch angles will be overestimated if differential energy flux is employed rather than distribution function, and this too reduces the apparent width of the $\mathrm{V}$. This point will be discussed further in section 5.3. Third, in section 5.3, we also point out that M\&B estimate of the distance to the cusp ion injection region, which for many ions (those that cross the magnetopause at larger elapsed times $t-t_{0}$ ) is closer to the satellite than the reconnection site; this too will lead to an underestimate of the width of the region (their equation (4)).

\subsection{Estimation of Reconnection Site From Energy- Pitch Angle Dispersion}

M\&B found that the cusp ion injection was at the magnetic cusp. This is not fully consistent with the reconnection model because, although it predicts that some ions come from the magnetic cusp (particularly the highest energy ions of a lower- $E_{i c}$ spectrum, for which $\left(t-t_{o}\right)$ is large), others (all ions of a high- $E_{i c}$ spectrum and the lower energy ions of a low- $E_{i c}$ spectrum, for which $\left(t-t_{0}\right)$ is smaller) are injected at earlier times at low magnetic latitudes. Hence by not reporting ion injection/acceleration at low latitudes the results of M\&B are inconsistent with a number of pieces of evidence that reconnection occurs at the subsolar magnetopause during periods of southward IMF: Gosling et al. [1990a] showed that opened field lines were moving away from the lowlatitude magnetopause in both hemispheres; Paschmann [1984] showed that the senses of the field RD defined by the stress balance test were opposite in the two hemispheres; the polarity of magnetopause FTE signatures is opposite in opposite hemispheres [Berchem and Russell, 1984; Rijnbeek et al., 1984]; streaming energetic ions show that FTEs at the northern/southern magnetopause are connected to the northern/southern hemisphere ionosphere, respectively [Daly et al., 1984]; Lockwood et al. [1993a] deduced an equatorial reconnection site from combined satellite and radar observations of cusp precipitation. Recently, Phillips et al. [1993] have also deduced low-latitude reconnection and injection from midaltitude particle observations by ISEE.

The method of M\&B is based on the folding of the pitch angle distributions as the particles precipitate into regions of higher magnetic field. Burch et al. $[1982,1986]$ and M\&B employed dipole and Olsen-Pfitzer field models. We here employ the last closed field line of a Tsyganenko T87 field model [Tsyganenko, 1987] for equinox conditions and $K p$ values of 2 and 6: these field lines are shown in Figure 13 (as a solid and dashed line, respectively). In each case an injection point at the subsolar magnetopause is marked by a solid circle, one at the magnetic cusp by a cross and one midway between the other two by an open circle. Figure 13 demonstrates that the distances from a midaltitude satellite to each of these points decreases with increasing $K p$ in this T87 model, where $K p$ here allows mainly for the effects of IMF $\mathrm{Bz}$ (via erosion) and of solar wind dynamic pressure (via compression). The dashed curve in Figure 14 shows the variation of magnetic field strength along this $K p=6$ field line, as a function of distance from a satellite at a geocentric height $r_{o}$ of $3.78 R_{E}$, chosen to model the DE 1 observations

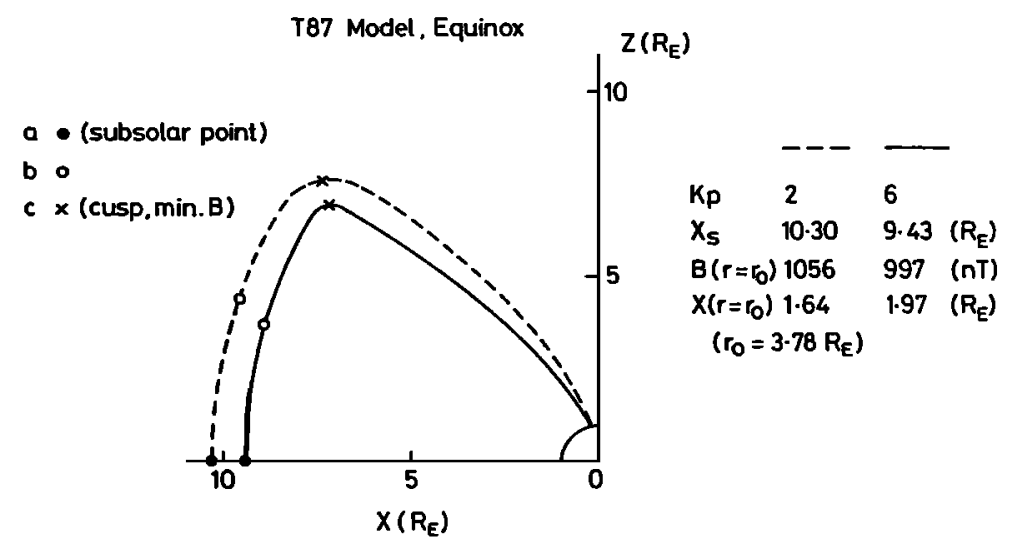

Figure 13. The sunwardmost field line of the Tsganyenko T87 magnetic field model in the GSE $X Z$ plane, for equinox conditions and $K p=2$ (dashed line) and 6 (solid line). The subsolar magnetopause is at GSE coordinates $\left(X_{s}, 0,0\right)$, where $X_{s}$ is $10.30 R_{E}$ for $K p=2$ and $9.43 R_{E}$ for $K p=6$. These model field lines are used to map pitch angles from the dayside magnetopause to a satellite at, or example, a geocentric distance of $r_{o}$ of $3.78 R_{E}$ where the field strength is $1056 \mathrm{nT}$ for $K p=2$ and $997 \mathrm{nT}$ for $K p=6$. The symbols give three injection points: the solid circle is the subsolar magnetopause, the cross is the magnetic cusp, and the open circle is halfway between them on the dayside magnetopause. 


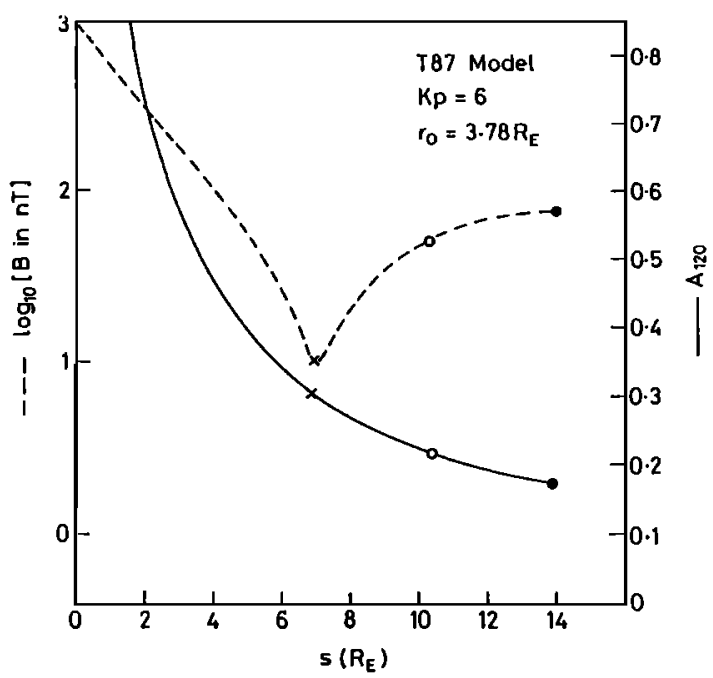

Figure 14. Parameters calculated for the T87 model, for the $K p=6$ model field line shown in Figure 13, plotted as a function of distance $S$ from a satellite at a geocentric distance of $r_{o}$ of $3.78 R_{E}$. The dashed curve shows the field strength $B$ showing a minimum at the magnetic cusp, the solid curve shows the $A_{120}$ parameter, which is a direct measure of the angle of the $V$ signature on logarithmic scale cusp ion spectrograms (see text). The symbols denote the locations shown in Figure 13.

presented by Lockwood and Smith (manuscript in preparation, 1994): the symbols are as in Figure 13. Note that the field has a minimum at the magnetic cusp.

As derived by Burch et al. [1982, 1986], adiabatic, scatterfree motion of an ion yields a relationship between its energy $E\left(\alpha_{s}\right)$ and its time of flight $\tau$ along a field-aligned distance from $z=S$ to the satellite at $z=0$ :

$$
E=\left(m / 2 \tau^{2}\right)\left\{\int_{0}^{S}\left[1-\sin ^{2} \alpha_{s}\left[B(z) / B_{s}\right]\right\}^{-1 / 2} d z\right\}^{2}
$$

where $\alpha_{s}$ and $B$, are the pitch angle and the field strength at the satellite and $m$ is the ion mass. Hence $E$ increases with $\alpha_{s}$ if $S$ and $\tau$ are constant. When looking at the angle of the resulting ion $V$ on a logarithmic spectrogram scale, it is useful to consider a quantity $A\left(\alpha_{s}\right)$ defined by

$$
A\left(\alpha_{s}\right)=\log _{10} E\left(\alpha_{s}\right)-\log _{10} E\left(\alpha_{s}=0\right)
$$

The parameter $A\left(\alpha_{s}\right)$ is a very useful one because it is a direct measure of the shape of the $V$ on the ion spectrogram, that shape being used to infer the injection distance. From (6) and (7)

$$
\left.A\left(\alpha_{s}\right)=2 \log _{10} \mid\left(\tau_{d} / \tau\right)\left[\int_{0}^{S}\left\{1-\sin ^{2} \alpha_{s}\left[B(z) / B_{s}\right]\right\}^{-1 / 2} d z\right] / S_{o}\right\}
$$

where $\tau_{o}$ is the time of flight of the zero pitch angle ions, for which the injection distance is $S_{0} . M \& B$, in fact, assumed that all ions in any one ion $\mathrm{V}$ were injected in the same time and place, and hence that $\tau=\tau_{0}$ and $S=S_{0}$. With this assumption we can compute the variation of $A$ for a given $\alpha_{s}$ with distance from the satellite $S$. The results are shown by the solid line in Figure 14 for the example of $\alpha_{s}=120^{\circ}$ $\left(A_{120}\right)$. Note that relatively small changes in the measured $A_{120}$ will cause large changes in the derived injection distance for

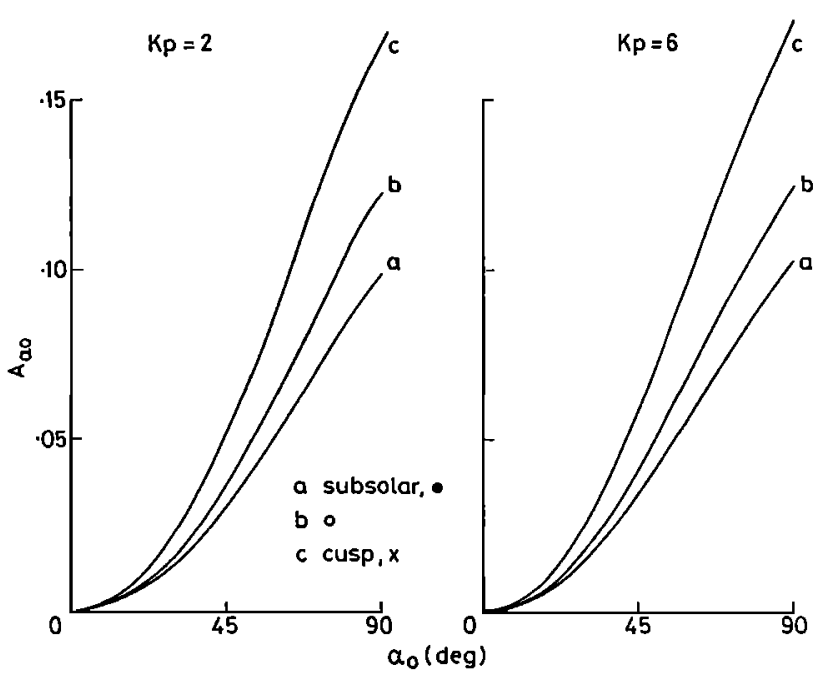

Figure 15. Shape of contours on a logarithmically scaled cusp ion spectrograms which link ions of constant time of flight $\tau$. The parameter $A$ is plotted as a function of $\alpha_{s}$ for the two field lines (left $K p=2$, right $K p u=6$ ), and the three marked injection points in Figure 13.

distances greater than the magnetic cusp. Specifically, $A_{120}$ falls from near unity at $S \approx 2 R_{E}$ to 0.3 near the magnetic cusp ( $S \approx 6 R_{E}$ for $K p=6$ model). However, if $S$ is increased further, $A_{120}$ only falls to 0.18 at the subsolar point $(S \approx$ $\left.14 R_{E}\right)$ : the gradient $\mathrm{d} A\left(\alpha_{\mathrm{s}}\right) / \mathrm{d} S$ being much smaller at the dayside magnetopause because the field strength $B$ increases with $S$. Hence a small change in the estimate of $A$ from an ion spectrogram causes large changes in the inferred injection distance.

Figure 15 shows the form of the full $A\left(\alpha_{s}\right)$ curves predicted for ions which were all injected at one time and point ( $\tau=\tau_{\mathrm{o}}$ and $S=S_{\mathrm{o}}$ ); that point being (a) subsolar, (b) at the midlatitude magnetopause, and (c) at the magnetic cusp (see Figure 13). As discussed by $M \& B$, the $V$ is flatter (smaller $\left.A\left(\alpha_{s}\right)\right)$ for more distant injection sites. The set of curves on the left is for the $K p=2$ version of the T87 model, and those on the right are for $K p=6$. It can be seen that the form of the curves is not sensitively dependent on the model field employed. This conclusion was reached by Burch et al. [1986], we here extend it to cover the Tsyganenko T87 field model.

The results shown in Figures 14 an 15 are essentially the same as presented by Burch et al. [1982, 1986] and used by M\&B. However, we wish to make a key point about the application of such results; namely, they must be applied to $E\left(\alpha_{s}\right)$ contours in each ion $\mathrm{V}$ for which the time of flight $\tau$ is constant. Because all ions in one $V$ share one observation time (to within one satellite spin period), this means that the $E\left(\alpha_{s}\right)$ contour used must join ions which were injected simultaneously. M\&B assume that all ions in an ion $V$ were injected simultaneously, and hence derive one injection distance for each ion V. However, they also compute a fieldperpendicular width of the injection region of roughly $1 R_{E}$, which corresponds to a distance of $\left(1 / \sin \theta_{s p}\right) \approx 10 R_{E}$ along the magnetopause. Hence low-energy ions were injected across the magnetopause from a point which is $\sim 10 R_{E}$ further away from the satellite than were the higher-energy ions seen at the same time and pitch angle. The idea that all ions in the 
$\mathrm{V}$ were injected simultaneously (and hence in the same location) and have the same time of flight (despite the range of energies observed at any one time and pitch angle) is inherent in M\&B's calculations and is evidenced by the fact that they ascribe a single injection distance to each ion $V$.

In order to understand the implications of this we can write, from equation (8):

$$
A\left(\alpha_{s}\right)=2 \log _{10}\left(\tau_{d} / \tau\right)+2 \log _{10}\left(S_{d} / S\right)+A^{\prime}\left(\alpha_{s}\right)
$$

where $A^{\prime}\left(\alpha_{s}\right)$ is the value derived if we assume that all ions are injected at the same time and place $\left(\tau_{o}=\tau, S_{o}=S\right)$. If we consider a contour in the ion $\mathrm{V}$, such that the field-aligned ions were injected at a later time (and hence as the field line evolves over the magnetopause at a smaller $S$ ) than those larger pitch angle ions which are observed simultaneously, equation (9) shows that $A\left(\alpha_{s}\right)$ will be smaller than $A^{\prime}\left(\alpha_{s}\right)$, with the result that the method of M\&B would tend to underestimate the distance to the injection point. Given that the distance inferred, $S$, depends sensitively upon $A\left(\alpha_{s}\right)$ (see Figure 14), this can cause considerable underestimation of $S$.

Consequently, in order to use the pitch angle-energy dispersion to estimate the injection location, it is vital that a contour of constant time of flight $\tau$ be used on the ion spectrogram. There is no reason why this should apply to either the average or the peak energy of an ion $V$ (at different $\alpha_{s}$ neither form a set which shares the same place and time of injection). However, this does apply to the minimum ion energy of the $V$, as at every $\alpha_{s}$ the minimum energy ions will have the longest time of flight and hence will have been the first injected (provided that minimum exceeds $E_{\min }$ at the $X$ line). Hence for all $\alpha_{s}$ the minimum energy ions were injected at the reconnection $X$ line and so the assumption that $\tau=\tau_{0}$ and $S=S_{0}$ can be employed. However, there is a problem in determining the minimum ion energy of the $\mathrm{V}$ when using spectrograms that display the differential energy flux. This problem is the subject of the remainder of this section.

In Figure 2 are shown four curves which join particles that would have the same (maximum) time of flight $\tau$ to the sarellite from an injection point at the $\mathrm{X}$-line considered. These are superposed on the "Cowley-D" distribution function for that injection point (see section 3). Also shown are the lines of constant pitch angle $\alpha_{s}$ at a midaltitude spacecraft at a geocentric height of $r_{0}=3.98 R_{E}$ (computed using the T87 model for $K p=6$ ). Each of the four curves intersects each $\alpha_{s}$ line twice (except that for $90^{\circ}$, which they only intersect tangentially): the higher-velocity intersection then refers to the greater of the two $\alpha_{s}$ values for each line. The four sets of curves are characterised by the ion energy observed at zero pitch angle $\left(\alpha_{s}=0\right)$, which is $E_{i c}$. As $\left(t_{s}-t_{o}\right)$ increases, $E_{c c}$ falls. Hence the four curves give the minimum energy ions arriving at the satellite at four different elapsed times since reconnection. If we apply Loiuville's theorem, following each of these curves yields the variation of the distribution function along the low-energy edge of the $\mathrm{V}$. This variation in $f(v)$ is shown as a function of pitch angle at the satellite, $\alpha_{s}$ in the top panel of Figure 16. Notice that the distribution function falls as we move to greater pitch angles. This fall is particularly marked for the lower $\left(t_{s}-t_{0}\right)$ case, that is, for the higher $E_{i c}$.

$M \& B$ scaled the variation of energy as function of $\alpha_{s}$ from spectrograms that showed contours of the differential energy

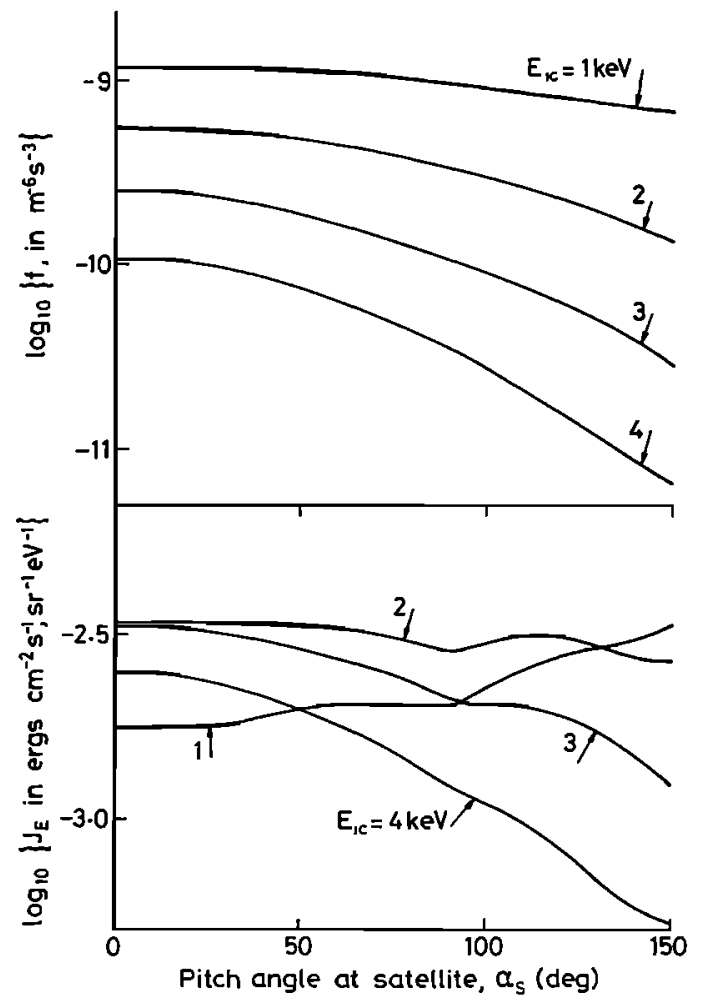

Figure 16. Variations (on logarithmic scales) of (top) distribution function $f$ and (bottom) differential energy flux $J_{E}$ as a function of observed pitch angle, $\alpha_{s}$, for minimumenergy ions for the injected distribution function shown in Figure 2. Curves are shown for four elapsed times since reconnection, each labeled by the minimum ion energy at zero pitch angle $\left(E_{a c}=1,2,3\right.$, and $\left.4 \mathrm{keV}\right)$

flux. Hence to evaluate the effect of the variation in $f(v)$ with $\alpha_{s}$ we present, in the lower part of Figure 16, the variation of the differential energy flux, $J_{E}(E)$, with $\alpha_{x}: J_{E}(E)$ being equal to $f(v)(2 / m) E_{c \alpha}{ }^{2}$, where $E_{c \alpha}$ is the lower cutoff ion energy detected at that time and pitch angle (note that $E_{u c}=$ $E_{c \alpha}\left(\alpha_{s}=0\right)$ ). Because they deal with the minimum ion energy (maximum time of flight), these curves are based purely on the recently-verified theory of the D-shaped distributions [Cowley, 1982] and on the time-of-flight from the reconnection site to the ionosphere (they do not also depend on the illustrative model of the evolution of the field line at subsequent times, as presented in section 4). They also assume that the magnetosheath ion temperature at the reconnection site was $2 \times 10^{6} \mathrm{~K}$.

Being the product of $f(v)$ and $E_{c a}{ }^{2}$, the behavior of $J_{E}\left(E_{c \alpha}\right)$ is complex. In addition to the variations of $f(v)$ shown in Figure 16a, the lower cut-off ion energy $E_{c \alpha}$ varies with pitch angle as given by Figure 15 and will also depend on the value of $E_{i c}$. For the large $E_{i c}$ case $(4 \mathrm{keV}), J_{E}\left(E_{c a}\right)$ falls by a factor of about 5 as $\alpha_{s}$ increases. This may be significant if the angle of the $V$ is scaled from an energy flux spectrogram. For such a spectrogram a contour of constant $J_{E}$ must have a steeper gradient with pitch angle than does the lowenergy cutoff energy, $E_{c \alpha}$. As shown by Figure 15, this gradient is also steeper if the particles are injected from closer to the satellite. Hence for this $E_{1 c}=4 \mathrm{keV}$ case the use of a constant $J_{E}$ contour will yield a distance which is 
smaller than the actual distance. The gradient of the solid curve in Figure 14 shows that this underestimation may be considerable. We conclude that the method of $M \& B$, applied to the minimum ion energies of the $V$, will underestimate the distance to the reconnection site (i.e., the injection site for the lowest-energy ions) for the most recently reconnected field lines (large $E_{i c}$ ). Figure $16 \mathrm{~b}$ shows that the fall in $J_{E}$ is smaller for $E_{i c}=3 \mathrm{keV}$, practically zero for $E_{t c}=2 \mathrm{keV}$ and is even a slight rise for the lowest $E_{i c}$ shown $(1 \mathrm{keV})$. This means that this effect decreases with $\left(t_{s}-t_{0}\right)$ and hence the underestimation of the injection distance derived for the minimum energy ions will also depend upon the location within the cusp from where the $V$ is observed.

To emphasize the points made in this section, we have taken the maximum and minimum field-aligned ion energy for the example V studied in detail by M\&B (their Figure 2). From this figure we find the maximum energy ions have an energy $E_{m t}=900 \mathrm{eV}$, whereas the minimum energy ions have energy $E_{x c}$ of about $200 \mathrm{eV}$. Even for the injection distance of $10 R_{E}$ derived by $M \& B$, these ions differ greatly in their time of flight, having values of 153 and $325 \mathrm{~s}$. In the interim period of $172 \mathrm{~s}$ the field line would have moved a distance of $5.4 R_{E}$ along the magnetopause for a de-Hoffman Teller frame velocity of $200 \mathrm{~km} \mathrm{~s}^{-1}$. This means that, to a first-order correction, the low energy ions have an injection distance of $(10+5.4)=15.4 R_{E}$ and a time of flight of $500 \mathrm{~s}$ : the interim period the must be revised to $(500-153)=347 \mathrm{~s}$ in which the field line moves $10.9 R_{E}$. Because the deHoffman Teller velocity values used here is low, this is a very conservative estimate of the width of the injection region, yet is greater than the field-perpendicular distance of $1 R_{E}$ computed by $M \& B$. Although the distance to the injection point derived by M\&B may be about right for higher-energy ions, it cannot also apply to the simultaneously-observed minimum energy ions.

We conclude that the midaltitude observations of M\&B are consistent with, rather than contradict, the idea that the cusp injection region is the entire dayside magnetopause and reconnection for southward IMF is at low latitudes.

\section{Discussion and Conclusions}

We have discussed the reconnection model of entry of magnetosheath plasma into the Earth's magnetosphere and ionosphere. The magnetosheath plasma crosses the boundary continuously along any field line for the entire period for which it is open. However, the precipitation we have come to term cusp is only seen in the ionosphere on any one field line for a limited interval of about $10 \mathrm{~min}$ after it is opened (section 4.3). Subsequently, and after the open field line has evolved into the tail lobe, the injected plasma is flowing antisunward at superthermal velocities in the mantle "RDexpansion fan" [Sanchez et al., 1990; Sanchez and Siscoe, 1990]: as a result, only the tail of the distribution of ions injected across the tail lobe boundary has an earthward directed field-parallel velocity and this forms the low-energy, low-density polar cap ion precipitation in the ionosphere. As the field line evolves from the dayside into the tail lobe, the precipitation evolves from cusp to polar cap via mantle. Before the arrival of cusp ions, time-of-flight effects will only allow fewer, higher-energy ions to precipitate to the ionosphere - a cleft precipitation on poleward-convecting open field lines. Newell and Meng [1991] consider the cleft population to be identical to that in the low-latitude boundary layer, and hence they use the terms interchangeably. The precipitation we refer to above as cleft originates from the open dayside LLBL and is the high-energy part of the Cowley-D population observed within the ion edge by, for example, Gosling et al. [1990b, c]. This does not preclude a second type of LLBL on closed field lines, particularly nearer the dawn and dusk flanks of the magnetosphere. In response to comments by Lockwood and Smith [1993a], Newell and Meng [1993] argue that convection is not directed from the LLBL to the cusp. However, inspection of their statistical maps shows that the precipitations they define as LLBL extend throughout the dayside, immediately equatorward of the cusp: hence it is not possible that there is not poleward convection through at least part of their LLBL and into the cusp. As pointed out by Lockwood and Smith [1993a], that part of the LLBL must be on open field lines, because of time-of-flight effects, and the precipitation will evolve into cusp with time elapsed since reconnection as the field line convect polewards. Some ions in this open LLBL population will show acceleration to above magnetosheath energies because of the acceleration at the dayside magnetopause, sunward of the magnetic cusp (where J.E $>0$ ).

The precipitation at the foot of a newly-reconnected field line therefore evolves from (open) LLBL, to cusp, to mantle to polar cap as the field line evolves into the tail lobe. This evolution will only be displayed in full on particle spectrograms for steady state conditions and a satellite path which is at all times aligned with the plasma flow streamlines. The above concepts were discussed by Cowley et al. [1991b] and Smith et al. [1992] and are consistent with joint satellite/radar measurements of transient reconnection events by Lockwood et al. [1993a] and by numerical simulations of steady state conditions [Onsager et al., 1993].

We have also discussed how the pulsating cusp model [Lockwood and Smith, 1989; Smith and Lockwood, 1990; Cowley et al., 1991b; Smith et al., 1992; Lockwood et al., 1993a] can explain jump features in the low-altitude cusp ion spectrograms in terms of pulses magnetic reconnection at the subsolar magnetopause. We have shown how these jumps are altered by the presence of a background level of reconnection between the pulses. The examples presented in the literature [Newell and Meng, 1992; Lockwood and Smith, 1992; Escoubet et al., 1992; Lockwood et al., 1993a] all show instantaneous jumps in the ions spectrogram, which reveal that the background reconnection rate between the pulses was zero and that there were large regions where the magnetopause was a tangential discontinuity (TD) between the patches of rotational discontinuity (RD). In section 3 we noted how such patches may be linked to magnetopause FTE signatures by the theory discussed by Saunders [1983], Southwood et al . [1988], Scholer [1988] and Semenov [1992].

From published examples of cusp ion spectrograms we conclude that both limits of the pulsating cusp model do occur; that is, quasi-steady reconnection and entirely pulsed reconnection. However, we also note that in this context, quasi-steady means that any variations in reconnection rate are less than a factor of about 2 over a period greater than about $10 \mathrm{~min}$.

The jumps predicted by the pulsating cusp theory for lowaltitude data may have one of a number of signatures at middle altitudes, depending on the relative motions of the satellite, the plasma and the open/closed boundary. The most 
common signature is likely to be a sawtooth signature of cusp ions spectrograms, with downward ramps of ion energy interrupted by upward jumps (Figure 10c). However, signatures similar to those at low altitudes could sometimes also be observed. If the satellite and convection speeds (normal to the open/closed boundary) are sufficiently well matched, the signature will be identical to that for steady state, independent of any reconnection rate variations.

For reconnection that does take place entirely in pulses, we have predicted the signatures that would be observed at mid altitudes. In particular, the jumps may show complex finite gyroradius effects if the boundary normal satellite and convection speeds are sufficiently similar. These finite gyroradius effects can result in double ion spectra being seen at a pitch angle away from zero, but only one will be seen for the field-parallel direction. The predictions presented here may have implications for the "double-injection" signatures recently reported by Woch and Lundin [1991, 1992a] from Viking data. From their published grey-scale spectrogram it is not clear if more than one population is seen in this example when the instrument is aligned with the magnetic field. If more than one population is present for field-aligned ions, then the signatures reported by Woch and Lundin are not a manifestation of pulsed reconnection at a single $X$ line and hence are unrelated to the jumps we describe here. In a companion paper (Lockwood and Smith, manuscript in preparation, 1994) we will discuss an example of the signature we have predicted here, as seen by the HAPI instrument on DE 1 , and we note that, unless the standard energy-time spectrogram is greatly expanded, it is not possible to see that there is only one population of fieldaligned ions at any one time. At least some of the examples of double injections in the Viking data also show only one field-aligned population at any one time (M. Yamauchi, private communication, 1993). It may be highly significant that double injections have not been reported in low-altitude observations, such as by DE 2 or DMSP, which are only of field-parallel ions. In the only example of such a double feature in low-altitude data of which we know, the second feature was due to helium ions that follow a second dispersion curve because of their greater mass (J.D. Winningham, private communication 1991). Hence the low altitude data also suggest that double injections may, in fact, be finite gyroradius effects around cusp ion jumps due to pulsed, single $\mathrm{X}$ line reconnection.

The apparent inconsistency between the reconnection particle-entry model and reported estimates of the width and location of the injection region can be explained by a number of factors, as discussed in sections 5.2 and 5.3, respectively. We conclude that the field perpendicular width of $\sim 1 R_{E}$ estimated for the injection region by $M \& B$ may be a slight underestimate but is substantially correct. We also agree with Burch et al. [1986] that the results do not depend critically upon the magnetic field model employed. The $1 R_{E}$ typically corresponds to a distance of $\sim 10 R_{E}$ along the magnetopause and hence if the higher $E\left(\alpha_{s}\right)$ ions of a V were injected from roughly $8 R_{E}$ from the Earth (as found by $M \& B$ ), the lower $E\left(\alpha_{s}\right)$ ions observed simultaneously were injected at about $18 R_{E}$, consistent with subsolar reconnection. We conclude that magnetosheath particles do enter the magnetosphere continuously, once a field line has been opened.

The density, temperature, and acceleration factor of the injected ions will vary as a function of elapsed time since reconnection $\left(t-t_{o}\right)$. In addition, time-of-flight effects complicate the spectrum of ions seen at low and middle altitudes. Those spectra will therefore also evolve as a function of $\left(t-t_{o}\right)$. It is not possible from the simple model described in section 4 to estimate the boundaries of (open) LLBL, cusp, mantle and polar cap precipitations, as would be defined by the criteria laid down by Newell and Meng [1991]. Such distinctions require full modeling of the complete spectrum. However, the example modelled by Onsager et al. [1993] shows a continuous evolution from one of these populations to another, as described conceptually by Cowley et al. [1991b]. This conclusion was also supported from combined satellite and ground-based observations by Lockwood et al. [1993a]. The jumps in the ion spectrum predicted by the pulsating cusp model will often mark a boundary between two of these regions by causing a change in the density and/or temperature across the thresholds that are used to define the regions. Examples of such cases have been presented by Lockwood et al. [1993a].

It is often wrongly assumed that pulsed reconnection leads to pulsed cusp precipitation [e.g., Newell and Sibeck, 1993]. This is not the case because of the prolonged period during which magnetosheath plasma is injected onto an open field line. In order to demonstrate this point we note that for the case presented by Lockwood et al. [1993a], the value of $E_{i c}$ has fallen to about $100 \mathrm{eV}$ at the poleward boundary of the cusp precipitation. From the combined radar and satellite data these authors show that the reconnection site was $16 R_{E}$ from the satellite. This means that the time of flight of these lowest energy cusp ions was $\left(t_{s}-t_{o}\right) \approx 12 \mathrm{~min}$. Hence in this case we could detect precipitation which we term cusp on a field line for at least $12 \mathrm{~min}$ after it is reconnected. However, such precipitation could not be seen at the foot of a field line until $\left(t_{s}-t_{o}\right) \approx 2 \mathrm{~min}$. Therefore we estimate that there is a period of at least $10 \mathrm{~min}$. during which cusp precipitation will be seen on any one newly-opened field line as it evolves into the tail lobe. Conversely, there would have to be a period of more than $10 \mathrm{~min}$. in which no reconnection took place at that MLT before the cusp precipitation would be entirely absent at low and middle altitudes (in such a case the previously opened flux has fully evolved into the tail lobe, before the subsequent pulse of reconnection occurs and in Figure $6 \mathrm{~b}$, the TD would cover most of the dayside magnetopause). We can gain an idea of how likely is such a prolonged period by looking at the statistics of the intervals between magnetopause FTEs. Figure 17 shows the cumulative probability distribution of inter-FTE intervals (for periods of southward IMF) from the statistical survey by Lockwood and Wild [1993]: $\tau$ is the repetition period of FTE signatures and $\delta t$ is their duration. This plot shows that the probability of an interval between two FTEs exceeding $10 \mathrm{~min}$ (the estimated time required for the cusp to vanish) is only 0.2 . This means that there is an $80 \%$ probability that the cusp will be observed, even if the reconnection only occurs in the short pulses which yield the FTEs. Similar arguments were presented by Smith et al. [1992], but based only on the mean repetition period of FTEs, not the full distribution, as presented here. This occurrence probability of the cusp is in good agreement with that derived from a statistical survey of low-altitude data [Newell et al., 1989].

This paper describes the complexity of cusp signatures that varying magnetopause conditions can cause. The width of the cusp trace on the ion spectrogram has been shown to depend 


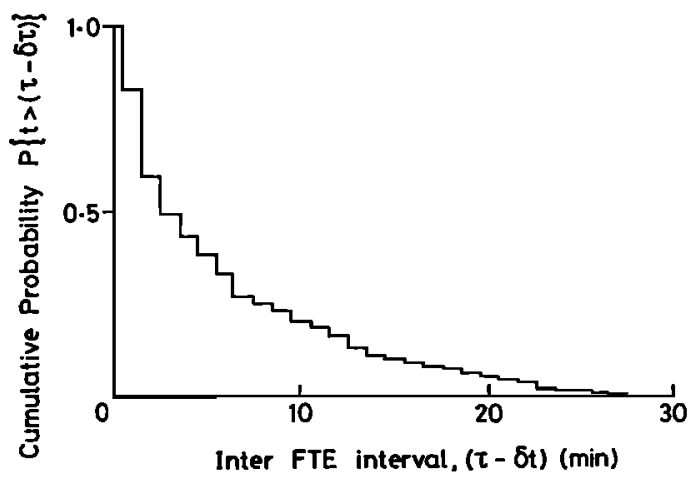

Figure 17. The cumulative probability distribution of the intervals between magnetopause FTE signatures: $\tau$ is the period between the centre of FTE signatures and $\delta t$ is the FTE duration, hence the interpulse interval is $(\tau-\delta t)$. The variable $P\{D(\tau-\delta t)\}$ is the probability that the interval between two FTE signatures exceeds the value $(\tau-\delta t)$ (adapted from Lockwood and Wild [1993]).

on the Alfven speed at the boundary, and further complications will result from the fact that this, in general, will vary with distance from the $X$ line (not included in section 4 ). The distribution of density and ion temperature in the magnetosheath will influence the precipitation characteristics and hence so will the upstream solar wind density, speed (and therefore dynamic pressure) and Mach number. In addition, the evolution of the newly opened field line will depend upon the orientation of the IMF and on the dipole tilt (i.e., on time of day and day of year). All such effects are relevant and will cause variations in the cusp precipitation. However, they can all be fitted into the theoretical framework of continuous entry of magnetosheath plasma along the open field lines produced by magnetic reconnection.

Last, it must be remembered that this paper has restricted its attention to southward IMF conditions, for which the reconnection site is at low latitudes on the dayside magnetopause. Much of the theory presented could be adapted to apply to injection caused by reconnection at the edge of the tail lobe during northward IMF. Under some circumstances (depending upon the IMF $B_{x}$ component and the time of year) one hemisphere may be shielded from either of these types of reconnection [Crooker, 1992; Knipp et al., 1993] in which case a "stagnant" cusp may be present [Jankowska et al., 1990; Lockwood, 1991b]. Neither dispersionless (stagnant) cusps nor V-shaped ion-energy dispersions [Reiff et al., 1980; Yamauchi et al., 1993] have been considered in this paper, given that they occur during northward IMF [Reiff et al., 1980].

Acknowledgments. The authors wish to thank S. W. H. Cowley, P. H. Reiff and T. G. Onsager for many valuable discussions of this work.

The Editor thanks T. G. Onsager and N. U. Crooker for their assistance in evaluating this paper.

\section{References}

Aggson, T.L., P.J. Gambardella, and N.C. Maynard, Electric field measurements at the magnetopause: 1 . Observations of large convective velocities at rotational magnetopause discontinuities, J. Geophys. Res., 88, 10,000, 1983.
Atkinson, G., Magnetospheric flows and substorms, in Mag netosphere-Ionosphere Interactions, edited by $\mathrm{K}$. Folkestadt, p. 203, Universitetsforlaget, Oslo, Norway, 1972.

Berchem, J., and C. T. Russell, Flux transfer events on the magnetopause: Spatial distribution and controlling factors, J. Geophys. Res., 89, 6689, 1984.

Biernat, H. K., M. F. Heyn, and V. S. Semenov, Unsteady Petschek reconnection, J. Geophys. Res., 92, 3392, 1987.

Burch, J.L. Rate of erosion of dayside magnetic flux based on a quantitative study of polar cusp latitude on the interplanetary magnetic field, Radio Sci., 8, 955, 1973.

Burch, J. L., Quasi-neutrality in the polar cusp, Geophys. Res. Lett., 12, 469, 1985.

Burch, J.L., J.D. Winningham, V.A. Blevins, N. Eaker, W.C. Gibson, and R.A. Hoffman, High-altitude plasma instrument for Dynamics Explorer-A, Space Sci. Instrum., 5, 455 , 1981.

Burch J.L, P.H. Reiff, R.A. Heelis, J.D. Winningham, W.B. Hanson, C. Gurgiolo, J.D. Menietti, R.A. Hoffman, and J.N. Barfield, Plasma injection and transport in the mid-altitude polar cusp, Geophys. Res. Lett., 9. 921, 1982.

Burch, J.L., et al., IMF By-dependent plasma flow and Birkeland currents in the dayside magnetosphere, 1, Dynamics Explorer observations , J. Geophys. Res., 90, 1577, 1985.

Burch, J. L., J. D. Menietti, and J. N. Barfield, DE-1 observa tions of solar wind-magnetospheric coupling processes in the polar cusp, in Solar Wind-Magnetosphere Coupling, edited by. Y. Kamide and J. A. Slavin, p. 441, Terra Scientific, Tokyo, 1986.

Candidi, M., G. Mastrantonio, S. Orsini, and C.-I. Meng, Evidence for the influence of the interplanetary magnetic field azimuthal component on the polar cusp configuration, J. Geophys. Res., 94, 13,585, 1989.

Carbary, J.F., and C.-I. Meng, Correlation of cusp width with $\mathrm{AE}(12$,$) and \mathrm{Bz}$, Planet. Space Sci., 36, 157, 1988.

Chapman, S., and V. C. A. Ferraro, A new theory of magnetic storms, 1, The initial phase (continued), J. Geophys. Res., $36,171,1931$.

Cowley, S.W.H., Magnetospheric asymmetries associated with the Y-component of the IMF. Planet Space Sci., 29, 79, 1981.

Cowley, S.W.H., The causes of convection in the Earth's magnetosphere: A review of developments during MS, Rev. Geophys., 20, 531, 1982.

Cowley, S.W.H. and M. Lockwood, Excitation and decay of solar-wind driven flows in the magnetosphere-ionosphere system, Ann. Geophys., 10, 103, 1992.

Cowley, S.W.H. and C.J. Owen, A simple illustrative model of open flux tube motion over the dayside magnetopause, Planet. Space Sci., 37, 1461, 1989.

Cowley, S.W.H., J.P. Morelli, and M. Lockwood, Dependence of convective flows and particle precipitation in the highlatitude dayside ionosphere on the $X$ and $Y$ components of the interplanetary magnetic field, J. Geophys. Res., 96, 5557,1991 a.

Cowley, S.W.H., M.P. Freeman, M. Lockwood, and M.F. Smith, The ionospheric signature of flux transfer events, in CLUSTER - dayside polar cusp, Eur. Space Agency Spec. Publ., $S P-330$, p. $1051991 \mathrm{~b}$.

Crooker, N.U., Reverse convection, J. Geophys. Res., 97, 19,363, 1992.

Crooker, N.U., F. Toffoletto, and M.S. Gusenhoven, Opening the cusp, J. Geophys. Res., 96, 3497-3503, 1991.

Daly, P.W., M. A. Saunders, R. P. Rijnbeek, N. Sckopke, and C. T. Russell, The distribution of reconnection geometry in flux transfer events using energetic ion, plasma and magnetic data, J. Greophys. Res., 89, 3843, 1984. 
Dungey, J.W., Waves and particles in the magnetosphere, in The Physics of the Magnetosphere, edited by R. L. Carovillano, J. F. McClay and H. R. Radoski, p. 246, D. Riedel, Norwell, Mass., 1968.

Elphic, R.C., Observations of flux transfer events: Are FTEs flux ropes, islands, or surface waves?, in Physics of Magnetic Flux Ropes, Geophys. Monogr. Ser., vol. 58, edited by C. T. Russell, E. R. Preist, and L. C. Lee, p. 455, AGU, Washington, D. C., 1990.

Escoubet, C.P., M.F. Smith, S.F. Fung, P.C. Anderson, R.A. Hoffman, E.M. Basinska, and J.M. Bosqued, Staircase ion signature in the polar cusp: a case study, Geophys. Res. Lett., 19, 1735, 1992.

Etemadi, A., S.W.H. Cowley, M. Lockwood, B.J.I. Bromage, D. M. Willis, and $\mathrm{H}$. Luhr, The dependence of high-latitude dayside ionospheric flows on the north-south component of the IMF, a high time resolution correlation analysis using EISCAT "POLAR" and AMPTE UKS and IRM data, Planet. Space Sci., 36, 471, 1988.

Farrugia, C.J., R.C. Elphic, D.J. Southwood, and S.W.H. Cowley, Field and flow perturbations outside the reconnected field line region in flux transfer events:theory, Planet. Space Sci., 35, 227, 1987a.

Farrugia, C.J., D.J. Southwood, S.W.H. Cowley, R.P. Rijnbeek, and P.W. Daly, Two-regime flux transfer events, Planet. Space Sci., 35, 737, 1987b.

Farrugia, C.J., et al., A multi-instrument study of flux transfer event structure, J. Geophys. Res., 93, 14,465, 1988.

Farrugia, C.J., M.P. Freeman, S.W.H. Cowley, D.J. Southwood, M. Lockwood, and A. Etemadi, Pressure-driven magnetopause motions and attendant response on the ground, Planet. Space Sci., 37, 589, 1989.

Foster, J.C., et al., Radar observations of cleft dynamics, $J$. Geophys. Res., 85, 3453, 1980.

Frank, L.A., Plasmas in the Earth's polar magnetosphere, $J$. Geophys. Res., 76, 5202, 1971.

Freeman, M.P. and D.J. South wood, The effects of magnetospheric erosion on mid- and high-latitude ionospheric flows, Planet. Space Sci., 36, 509, 1988.

Fuselier, S.A., D.M. Klumpar, and E.G. Shelley, Ion reflection and transmission during reconnection at the Earth's subsolar magnetopause, Geophys. Res. Lett., 18, 139, 1991.

Gosling, J.T., M.F. Thomsen, S.J. Bame, R.C. Elphic, and C.T. Russell, Plasma flow reversals at the dayside magnetopause and the origin of asymmetric polar cap convection, $J$. Geophys. Res., 95, 8073, 1990a.

Gosling, J.T., M.F. Thomsen, S.J. Bame, T.G. Onsanger, and C.T. Russell, The electron edge of the low-latitude boundary layer during accelerated flow events. Geophys. Res. Lett., 17, 1833, 1990b.

Gosling, J.T., M.F. Thomsen, S.J. Bame, R.C. Elphic, and C.T. Russell, Cold ion beams in the low-latitude boundary layer during accelerated flow events, Geophys. Res. Lett., 17, 2245, 1990c.

Haerendel, G., G. Paschmann, N. Sckopke, H. Rosenbauer, and P.C. Hedgecock, The frontside boundary layer of the magnetopause and the problem of reconnection, $J$. Geophys. Res., 83, 3195, 1978.

Hapgood, M.A., and M. Lockwood, On the voltage and distance across the low-latitude boundary layer, Geophys. Res. Lett., $20,145,1993$.

Hapgood, M.A., Y. Tulunay, M. Lockwood, G. Bowe, and D.M. Willis, Variability of the interplanetary medium at $1 \mathrm{AU}$ over 24 years: 1963-1986, Planet. Space Sci., 39, 411, 1991.

Heikkila, W. J., The morphology of auroral particle precipitation in Space Research 12, p. 1343, Akademie-Verlag, Berlin, 1972.
Heikkila, W.J., and J. D. Winningham, Penetration of magnetosheath plasma to low altitudes through the dayside magnetosheric cusps, J. Geophys. Res., 76, 883, 1971.

Hill, T.W., Rates of mass, momentum, and energy transfer at the magnetopause, in Proceedings of the Magnetospheric Boundary Layers Conference, Alpbach, Eur. Space Agency Publ., SP-148, p. 325, 1979.

Hill, T.W., and P.H. Reiff, Evidence of magnetospheric cusp proton acceleration by magnetic merging at the dayside magnetopause, J. Geophys. Res., 82, 3623, 1977.

Horwitz, J.L., and S.-I. Akasofu, The response of the dayside aurora to sharp northward and southward transitions of the interplanetary magnetic field and to magnetospheric substorms, J. Geophys. Res., 82, 2723, 1977.

Jankowska, K., et al., The configuration of the auroral distribu tion for interplanetary magnetic field $\mathrm{Bz}$ northward. 2 . Ionospheric convection consistent with VIKING observations, J. Geophys. Res., 95, 5805, 1990.

Jørgenson, T.S., E. Friis-Christensen, and J. Wilhjelm, Interplanetary magnetic direction and high latitude ionospheric currents, J. Geophys. Res., 77, 1976, 1972.

Knipp, D.J., et al., Ionospheric convection response to a magnetic cloud II: A case study for January 14, 1988. J. Geophys. Res., 98, 19,273, 1993.

Lee, L.C., and Z.F. Fu, A theory of magnetic flux transfer at the Earth's magnetopause, Geophys. Res. Lett., 12, 105, 1985.

Lockwood, M., Flux Transfer Events at the dayside magnetopause: Transient reconnection or magnetosheath pressure pulses?, J. Geophys. Res., 96, 5497, 1991.

Lockwood, M., Incoherent scatter radar measurements of the cusp in CLUSTER - dayside polar cusp, Eur. Space Agency Publ., SP-330 p. 57, 1991.

Lockwood, M. and S.W.H. Cowley, Ionospheric convection and the substorm cycle, in Substorms 1, Eur. Space Agency Publ., SP-335, 99, 1992.

Lockwood, M., and M.F. Smith, Low altitude signatures of the cusp and flux transfer events, Geophys. Res. Lett., I6, 879, 1989.

Lockwood, M., and M.F. Smith, Reply to Newell, Geophys. Res. Lett., I7, 305, 1990.

Lockwood, M., and M.F. Smith, The variation of reconnection rate at the dayside magnetopause and cusp ion precipitation, J. Geophys. Res., 97, 14,841, 1992.

Lockwood, M., and M.F. Smith, Comment on "Mapping the dayside ionosphere to the magnetosphere according to particle precipitation characteristics" by Newell and Meng, Geophys. Res. Lett., 20, 1739, 1993.

Lockwood, M., and M.N. Wild, On the quasi-periodic nature of magnetopause flux transfer events, J. Geophys. Res., 98, 5935, 1993.

Lockwood, M., S.W.H. Cowley, and M.P. Freeman, The excitation of plasma convection in the high latitude ionosphere, J. Geophys. Res., 95, 7961, 1990.

Lockwood, M, W.F. Denig, A.D. Farmer, V.N. Davda, S.W.H. Cowley, and H. Lühr, Ionospheric signatures of pulsed magnetic reconnection at the Earth's magnetopause, Nature, $361,(6411), 424,1993 a$.

Lockwood, M., J. Moen, S.W.H. Cowley, A.D. Farmer, U.P. Løvhaug, H. Luhr, and V.N. Davda, Variability of dayside convection and motions of the cusp/cleft aurora, Geophys. Res. Lett., 20, 1011, 1993b.

Lockwood, M., H.C. Carlson and P.E. Sandholt, The implica tions of the altitude of transient $630 \mathrm{~nm}$ dayside auroral emissions, J. Geophys. Res., 98, 15,571, 1993c.

Maynard, N.C., T.L. Aggson, E.M. Basinska, W.J. Burke, P. Craven, W.K. Peterson, M. Sugiura, and D. Weimer, Magnetospheric boundary dynamics: DE 1 and DE 2 observations near the magnetopause and the cusp, $J$. Geophys. Res., 96, 3505, 1991. 
Menietti, J.D., and J.L. Burch, Spatial extent of the plasma injection region in the cusp-magnetosheath interface, $J$. Geophys. Res., 93, 105, 1988

Newell, P.T., Comment, Geophys. Res. Lett., 17, 303, 1990.

Newell, P. T. and C.-I. Meng, The cusp and the cleft/LLBL: Low altitude identification and statistical local time variation, J. Geophys. Res., 93, 14,549, 1988.

Newell, P.T. and C.-I. Meng, On quantifying the distinctions between the cusp and the cleft/LLBL, in Electromagnetic Coupling in the Polar Clefis and Caps, NATO ASI Ser. C, vol. 278, edited by P.E. Sandholt and A. Egeland, p. 87, Kluwer Academic, Hingham, Mass., 1989.

Newell, P.T., and C.-I. Meng, Ion acceleration at the equatorward edge of the cusp: low altitude observations of patchy merging, Geophys. Res. Lett, 18, 1829, 1991.

Newell, P.T. and C.-I. Meng, Mapping the dayside ionosphere to the magnetosphere according to particle precipitation characteristics, Geophys. Res. Lett., 19, 609, 1992.

Newell, P.T., and C.-I. Meng, Reply, Geophys. Res. Lett., I6, $1741,1993$.

Newell, P.N. and D.G. Sibeck, $B_{y}$ fluctuations in the magnetosheath and azimuthal flow velocity transients in the dayside ionosphere, Geophys. Res. Lett., 20, 1719, 1993.

Newell, P.T.,C.-I. Meng, D.G. Sibeck, and R.P. Lepping, Some low-altitude cusp dependencies on the interplanetary magnetic field, J. Geophys. Res., 94, 8921, 1989.

Onsager, T.G., C.A. Kletzing, J.B. Austin, and H. MacKiernan, Model of magnetosheath plasma in the magnetosphere: cusp and mantle precipitations at low altitudes, Geophys. Res. Lett., 20, 479, 1993.

Paschmann, G., The Earth's magnetopause, in Achievements of the International Magnetospheric Study, IMS, Eur. Space Agency Publ. SP-217, p 53-64, 1984.

Paschmann, G., Comment on "Electric field measurements at the magnetopause, 1 , Observation of large convective velocities at rotational magnetopause discontinuities" by T.L. Aggson, P.J. Gambardella, and N.C. Maynard, J. Geophys. Res., 90, 7629, 1985.

Paschmann, G., et al., Plasma acceleration at the Earth's magnetopause: Evidence for reconnection, Nature, 282, $243,1979$.

Paschmann, G., G. Haerendel, I. Papamastorakis, N. Sckopke, S. J. Bame, J. T. Gosling, and C.T. Russell, Plasma and magnetic field characteristics of magnetic flux transfer events, J. Geophys. Res., 87, 2159, 1982.

Paschmann, G., I. Papamastorakis, W. Baumjohann, N. Sckopke, C.W. Carlson, B.U.Ö. Sonnerup, and H, Luhr, The magnetopause for large magnetic shear: AMPTE/IRM observations, J. Geophys. Res., 91, 11,099, 1986.

Paschmann, G., B.U.Ö. Sonnerup, I. Papamastorakis, W. Baumjohann, N. Sckopke, and H, Luhr, The magnetopause and boundary layer for small magnetic shear: convection electric fields and reconnection, Geophys. Res. Lett., 17, $1829,1990$.

Phillips, J.L., S.J. Bame, R.C. Elphic, J.T. Gosling, M.F. Thomsen, and T.G. (Onsager, Well-resolvd observations by ISEE 2 of ion dispersion in the magnetospheric cusp, $J$. Geophys. Res., 98, 13,429, 1993.

Reiff, P.H., T.W. Hill, and J.L. Burch, Solar wind plasma injection at the dayside magnetospheric cusp, $J$. Geophys. Res., 82, 479, 1977.

Reiff, P.H., J.L. Burch, and R.W. Spiro, Cusp proton signatures and the interplanetary magnetic field, J. Geophys. Res., 85, 5997, 1980.

Rijnbeek, R.P., S.W.H. Cowley, D.J. Southwood, and C.T. Russell, A survey of dayside flux transfer events observed by the ISEE 1 and 2 magnetometers, J. Geophys. Res., 89, $786,1984$.
Rosenbauer, H., H. Gruenwaldt, M.D. Montgomery, G. Paschmann, and N. Sckopke, HEOS-2 plasma observations in the distant polar magnetosphere: the plasma mantle, $J$. Geophys. Res., 80, 2723, 1975.

Russell, C.T., and R.C. Elphic, Initial ISEE magnetometer results: Magnetopause observations, Space Sci. Rev., 22. $681,1978$.

Russell, C.T., and R.C. Elphic, ISEE observations of flux transfer events at the dayside magnetopause, Geophys. Res. Lett., 6, 33, 1979.

Sanchez, E.R. and G.L. Siscoe, IMP 8 magnetotail boundary crossings: a test of the MHD models for an open magnetosphere, J. Geophys. Res., 95, 20,771, 1990.

Sanchez, E.R., G.L. Siscoe, J.T. Gosling, E.W. Hones, Jr., and R.P. Lepping, Observations of rotational discontinuity-slow mode expansion fan structure of the magnetotail boundary, J. Geophys. Res., 95, 61, 1990.

Saunders, M.A., Recent ISEE observations of the magnetopause and low-latitude boundary layer: A review, J. Geophys., 52, $190,1983$.

Saunders, M.A., C.T. Russell, and N. Sckopke, A dual-satellite study of spatial properties of FTEs, in Magnetic Reconnection in Space and Laboratory Plasmas, Geophys. Monogr. Ser., vol. 30, edited by E. W. Hones, Jr., p. 145, AGU, Washington, D. C., 1984.

Saunders, M.A., M.P. Freeman, D.J. Southwood, S.W.H. Cowley, M. Lockwood, J.C. Samson, C.J. Farrugia, and T.J. Hughes, Dayside ionospheric convection changes in response to long period IMF oscillations: determination of the ionospheric phase velocity, J. Geophys. Res., 97. 19373, 1992.

Scholer, M., Magnetic flux transfer at the magnetopause based on single X-line bursty reconnection, Geophys. Res. Lett., IS, 291, 1988.

Semenov, V.S., I.V. Kubyshkin, V.V. Lebedeva, M.V. Sidneva, H.K. Biernat, M.F. Heyn, B.P. Besser, and R.P. Rijnbeek, Time-dependent localized reconnection of skewed magnetic fields, J. Geophys. Res., 97, 4251, 1992.

Sibeck, D. G., A model for the transient magnetospheric response to sudden solar wind dynamic pressure variations, J. Geophys. Res., 95, 3755, 1990.

Siscoe, G.L., The magnetosphere boundary, in Physics of Space Plasmas, SPI Conf. Proc. Reprint Ser., vol. 7, p. 3, Scientific, Cambridge, Mass., 1987.

Siscoe, G.L. and T.S. Huang, Polar cap inflation and deflation, J. Geophys. Res., 90, 543, 1985.

Smith, M.F. and M. Lockwood, The pulsating cusp, Geophys. Res. Lett., 17, 1069, 1990.

Smith, M.F. and Rodgers, D.J., Ion distributions at the dayside magnetopause, J. Geophys. Res., 95, 11,617, 1991.

Smith, M.F., M. Lockwood, and S.W.H. Cowley, The statistical cusp: a simple flux transfer event model, Planet. Space Sci., 40, 1251, 1992.

Sonnerup, B.U.Ö., G. Paschmann, I. Papamastorakis, N. Sckopke, G. Haerendel, S.J. Bame, J.R. Ashbridge, J.T. Gosling, and C.T. Russell, Evidence for magnetic field reconnection at the Earth's magnetopause, J. Geophys. Res., $86,10,049,1981$.

Sonnerup, B.U.Ö., I. Papamastorakis, G. Paschmann, and H, Luihr, The magnetopause for large magnetic shear: analysis of convection electric fields from AMPTE/IRM, $J$. Geophys. Res., 95, 10,541, 1990.

Southwood, D.J., Theoretical aspects of ionosphere, magnetosphere-solar wind coupling, Adv. Space Res., 5, (4), 7, 1985.

Southwood, D.J., The ionospheric signature of flux transfer events, J. Geophys. Res., 92, 3207, 1987.

Southwood, D.J., M.A. Saunders, M.W. Dunlop, W.A.C. Mier- 
Jedrzejowicz, and R.P. Rijnbeek, A survey of flux transfer events recorded by UKS spacecraft magnetometer, Planet. Space Sci., 34, 349, 1986.

Southwood, D.J., C.J. Farrugia, and M.A. Saunders, What are flux transfer events?, Planet. Space Sci., 36, 503, 1988.

Spreiter, J.R., A.L. Summers, and A.Y. Alksne, Hydromagnetic flow around the magnetosphere, Plaket. Space Sci., I4, $223,1966$.

Taguchi, S., M. Sugiura, J.D. Winningham, and J.A. Slavin, Characterization of the IMF $B_{y}$ dependent field-aligned currents in the cleft region based on DE 2 observations, $J$. Geophys. Res., 98, 1393, 1993.

Todd, H., S.W.H. Cowley, M. Lockwood, D.M. Willis, and H. Luthr, Response time of the high-latitude dayside ionosphere to sudden changes in the north-south, component of the IMF, Planet. Space Sci., 36, 1415, 1988.

Tsyganenko, N.A., Global quantitative models of the geomag netic field in the cislunar magnetosphere for different disturbance levels, Planet. Space Sci., 35, 1347, 1987.

Winningham, J.D., J.L Burch, N. Eaker, V.A. Blevins, and R.A. Hoffman, The low altitude plasma instrument (LAPI), Space Sci. Instrum., 5, 465, 1981.
Woch, J., and R. Lundin, Temporal magnetosheath plasma injection observed with Viking: a case study, Ann. Geophys., 9, 133, 1991.

Woch, J., and R. Lundin, Signatures of transient boundary layer processes observed with Viking, J. Geophys. Res., 97. 1431, 1992a.

Woch, J., and R. Lundin, Magnetosheath plasma precipitation in the polar cusp and its control by the interplanetary magnetic field, J. Geophys. Res., 97, 1421, 1992b.

Yamauchi, M., J. Woch, R. Lundin, M. Shapshak and R. Elphinstone, A new type of ion injection event observed by Viking, Geophys. Res. Lett., 20, 795, 1993.

M. Lockwood, Rutherford Appleton Laboratory, Chilton, Didcot, OX11 OQX, UK. (e-mail: Internet. mike@eiscat.ag.rl.ac.uk)

M. F. Smith, Laboratory for Extraterrestrial Physics, NASA Goddard Space Flight Center, Greenbelt, MD 20771. (e-mail: Internet. smith@lepmfs.gsfc.nasa.gov)

(Received June 28, 1993; revised September 28, 1993; accepted November 22, 1993.) 